\title{
Presencia e impacto espacial de los sectores creativos en las zonas metropolitanas de México*
}

\section{Spacial economic impacts of creative industries of the metropolitan areas of Mexico}

Marcos Valdivia López**

Resumen

La dinámica de crecimiento de las ciudades contemporáneas y su estructura territorial están influidas por las actividades económicas asociadas a las industrias o sectores creativos. Se realiza un análisis espacial de las actividades económicas creativas en todas las zonas metropolitanas (ZM) de México. Para mostrar la importancia espacial que las industrias creativas tienen sobre la estructura urbana del país, se calcula la concentración del empleo de cada uno de los sectores y subsectores económicos respecto de la distancia con el centro económico de la ZM más cercana. Concluimos que las industrias creativas tienden a localizarse en el centro económico de las zM y son, entre todas las actividades económicas, de las que tienen mayor capacidad para desarrollar derramas positivas en el crecimiento del empleo.

Palabras clave: clase creativa, industrias creativas, terciarización, derramas, externalidades urbanas.
Abstract

Growth dynamics in contemporary cities and their territorial organization are subject to the influence of economic activities associated with creative industries. In this research, we perform a spatial analysis of such creative industries across all metropolitan areas of Mexico during the period. In order to show the spatial relevance that creative industries have over the urban structure, we calculate the employment concentration of each of the sectors and subsectors with respect to the distance from the closest metropolitan area's Central Business District. We conclude that creative industries tend to localize close to the Metropolitan Areas' CBD and they are the ones with greater capacity to generate positive externalities on employment growth.

Keywords: creative class, creative industries, tertiaritation, spillovers, urban externalities.

Recibido el 8 de octubre de 2013.

Aprobado el 31 de julio de 2014.

* El autor agradece el apoyo técnico de Nelly Linares y la lectura de Carlos Galindo de una versión preliminar del documento. Asimismo, se agradecen las sugerencias de dos dictaminadores anónimos quienes ayudaron a precisar y mejorar el escrito final.

** Investigador del Programa de Estudios Regionales del Centro Regional de Investigaciones Multidisciplinarias de la Universidad Nacional Autónoma de México. Correo electrónico: marcosv@correo.crim.unam.mx 


\section{Introducción}

Hoy en día el crecimiento de las ciudades no puede entenderse sin considerar el efecto que el capital humano tiene sobre éstas. La investigación reciente en el campo de la economía urbana postula en general que el éxito económico alcanzado por las ciudades se explica, en buena medida, por la presencia de población calificada y por la capacidad de las ciudades para atraer más gente calificada (Glaeser, 2011). Al respecto, algunos autores en boga en los estudios urbanos como Richard Florida (2012) han argumentado que la existencia de oportunidades de empleo con buenos salarios no es una condición suficiente para atraer migrantes calificados a las ciudades, sino que además es necesario que éstas ofrezcan un estilo de vida y consumo que sea atractivo.

Lo anterior nos lleva a plantear si alguna de las ideas anteriormente señaladas podrían ser útiles para entender la dinámica de crecimiento de las ciudades mexicanas, en particular la de sus zonas metropolitanas (ZM). En esta investigación analizamos si la dinámica de crecimiento de las zonas metropolitanas está condicionada o no por la presencia de una clase o sector creativo. En particular, proponemos que algunas de las transformaciones más importantes que ha tenido el país en materia de estructura urbana como lo son la consolidación de zM medias exitosas (Querétaro) y la transformación económica (terciarización) de las grandes metrópolis como la zm de la Ciudad de México y la de Guadalajara han dependido de la consolidación y presencia de un sector creativo al interior de las mismas que tiene la capacidad de atraer migración calificada.

Para fundamentar lo anterior, en este trabajo analizamos la dinámica de crecimiento de las 56 zm del país (Sedesol, Conapo, INEGi, 2007), a partir de la evaluación de los sectores económicos que están asociados a lo que se ha denominado como "clase creativa" (Florida, 2005). La propuesta metodológica consiste, en primer lugar, en detectar y medir a un sector económico creativo al interior de las zM, y posteriormente evaluar el papel que éste podría estar desempeñando en la estructura espacial de empleo y en el crecimiento de las zonas metropolitanas.

$\mathrm{Al}$ resto del documento le quedan seis secciones. En la sección dos, se discuten algunos antecedentes teóricos sobre la clase creativa y el capital humano. En la sección tres, se propone una clasificación del sector 
creativo a partir de los censos económicos y se detalla la presencia que este sector tiene en las 56 zM del país. En la sección cuatro, se analiza la concentración del empleo - a partir de regresiones de gradiente de empleo- de cada uno los subsectores económicos a nivel de Área Geoestadística Básica (AGEB) y se discute el grado de centralidad de los subsectores clasificados como creativos. En la sección cinco, se analiza un modelo simple de convergencia condicional para tener un primer acercamiento al impacto del sector creativo en el crecimiento de las zM. En la sección seis, se analiza si el sector creativo genera externalidades en la creación de empleo al interior de las ciudades. En la sección siete, se identifican las aglomeraciones espaciales a nivel de AGEB del sector 54 en las ZM con mayor presencia de actividades creativas. El documento concluye con una sección de comentarios finales.

\section{Antecedentes teóricos}

La noción de clase creativa está fundamentalmente asociada a la publicación del célebre libro de Richard Florida The Rise of Creative Class (2012), en donde se argumenta que el crecimiento económico actual no sólo depende del uso intensivo de las nuevas tecnologías y del internet, sino principalmente de la concentración de gente talentosa o creativa en las ciudades. Esta idea en realidad no es del todo nueva para un lector familiarizado con la teoría del capital humano en el campo de la economía urbana. Glaeser (2005) sostiene que la idea de clase creativa es sólo un término rimbombante para argumentar que el crecimiento de las ciudades será mayor a mayor presencia de capital humano en las mismas, así como de su capacidad para ofrecer estilos de consumo atractivos que atraigan y retengan más capital humano.

Independientemente de si el concepto y la noción apropiada es clase creativa o capital humano, la idea relevante en nuestra investigación es destacar que esta polémica conlleva una discusión sobre las decisiones de localización de gente calificada, en función de una serie de atributos del ambiente urbano (o "amenidades").

Storper y Scott (2009) delinean las implicaciones que tiene la discusión de la clase creativa (o capital humano) para la literatura urbana y regional. 
Estos autores señalan que la disyuntiva que ha guiado las teorías de crecimiento urbano desde Weber hasta Alonso y, recientemente, la "nueva goegrafía económica” à la Krugman, ha sido saber si la gente sigue a las firmas o las firmas siguen a la gente. Las teorías de la clase creativa y/o del capital humano, al privilegiar las decisiones de localización de los individuos en relación con los valores de las amenidades urbanas, estarían más del lado argumental que señala que las "firmas siguen a los trabajadores". A decir de Storper y Scott, una de las principales críticas a esto es que deja a un lado los aspectos dinámicos de la geografía de la producción y el trabajo en el crecimiento urbano; es decir, en las ciudades hay economías de aglomeración que refuerzan de manera circular y acumulativa el crecimiento (2009, p. 153). En este sentido, una visión más amplia de lo que debiera ser una teoría de la clase creativa debe incluir en la decisión de localización de los individuos, además de las amenidades, las oportunidades de empleo y salarios en las ciudades. ${ }^{1}$

Siendo justos, la teoría de la clase creativa (à la Florida) y la de capital humano (à la Glaeser) mantienen entre sí importantes diferencias que no deben ser soslayadas. Como señalan Boschma y Fritsch (2009), la visión de clase creativa abandona la perspectiva sectorial y se concentra en las ocupaciones (ya que puede haber gente talentosa en cualquier industria), mientras que la visión del capital humano descansa más en las externalidades de aglomeración que pueden ser tipo Arrow-Marshall-Romer (inter-firmas) o tipo Jacobs (intra-firmas). En particular, las economías de aglomeración producen derramas (spillovers) de conocimiento entre firmas e industrias, mientras que las derramas de la clase creativa son producidas entre personas que interactúan en una ciudad o región (Brakman, Garretse y

${ }^{1}$ En lo que se ha denominado un "modelo de equilibrio espacial" puede encontrarse la versión moderna de una teoría de localización de individuos à la Alonso al interior de una ciudad (Glaeser, 2008). En este modelo, la localización de un individuo se puede explicar en su forma más general a partir de la siguiente relación lineal: ingresos + amenidades - costos de transporte - costos de vivienda. En este marco, se argumenta que hay un equilibrio espacial ya que el flujo de utilidad que otorgan estas variables a un individuo en su decisión de localización es constante a lo largo del espacio; es decir, una vez que se alcance dicho estado, ya no hay incentivo (o utilidad o ganancia extra por obtener) debido a un cambio de lugar o localización. En el marco de nuestra discusión, el modelo anterior sugeriría que la variable de "amenidades" sería aquella con mayor efecto sobre las decisiones de localización de la "clase creativa". 
Marrewijk, 2009, p. 277). Una implicación de esto es que la clase creativa por su naturaleza tenderá a concentrarse en las cercanías del centro o centros económicos de una ciudad o en barrios (gentrificados) donde las externalidades generadas sean producto del intercambio de ideas en un área que concentra gente talentosa.

Un punto importante en esta discusión es cómo medir la clase creativa. En principio, la clase creativa debería ser clasificada a partir de profesiones (u ocupaciones) y no por niveles de educación ${ }^{2}$ o afiliaciones industriales. Al respecto, Florida (2012) originalmente propone tres sectores ocupacionales para la identificación de la clase creativa: un grupo duro de creativos (científicos de ciencias duras y sociales, médicos, profesores, ingenieros, arquitectos, etc.); un grupo bohemio (artistas, escritores, fotógrafos, diseñadores, etc.); y un grupo ocupacional de profesiones creativas (ocupaciones asociadas a las finanzas y administración de empresas, abogados, bienes raíces, miembros de la clase política, etc.). Diversos estudios empíricos pueden encontrarse en la literatura que utilizan esta clasificación (por ejemplo, Boschma y Fritsch, 2009, p. 396). Ademas, Florida, Mellander, Solarick y Ross (2012), en un trabajo reciente que explica la dispersión salarial entre zonas metropolitanas en Estados Unidos, proponen una clasificación ocupacional donde además se distinguen calificaciones analíticas, inteligencia social y calificación física. ${ }^{3}$

Asimismo, la literatura empírica reporta otro grupo de estudios que adoptan una clasificación sectorial para analizar a la clase creativa; en éstos, básicamente se clasifican sectores creativos con base en su actividad

${ }^{2}$ Glaeser (2005) indica que las ocupaciones creativas en realidad son medidas de calificación (educación) y no creatividad.

${ }^{3}$ Es importante señalar que este tipo de clasificaciones ocupacionales de la clase creativa no está exenta de crítica; en particular, se ha aludido que los subgrupos ocupacionales utilizados incluyen a un sector rentista y especulativo (asociado a las profesiones creativas involucradas en las finanzas y los bienes raíces -o the dealer class-) que no necesariamente es productivo y que puede incluso inhibir el desarrollo regional, tal como la reciente crisis financiera mundial del 2009 evidenció con las prácticas perniciosas que sectores profesionales asociados al sector financiero o de bienes raíces desarrollaron a partir del uso y manejo de "nuevos instrumentos financieros" con fines especulativos (Krätke, 2010). Adicionalmente, puede señalarse que la noción de "clase" descansa en una tradición sociológica basada en ocupaciones (o estatus social) que no considera elementos distribucionales del ingreso o la renta. 
industrial. Por ejemplo, Fingleton, Igliori, Moore y Odedra (2007) clasifican actividades industriales distinguiendo industrias creativas y técnicas del resto de actividades; Martin-Brelot, Grossetti, Eckert, Gritsai y Kovács (2010) analizan la movilidad espacial de la clase creativa en Europa y de manera similar también distinguen industrias de conocimiento intensivo (ingeniería, química) e industrias creativas (diseño, arquitectura, software, videojuegos) del resto de actividades. Este último enfoque tiene la ventaja de que además toma en cuenta las externalidades que podrían darse entre firmas o sectores, y de esa manera incorporar la crítica que realizan Storper y Scott (2009) de que la clase creativa tiene la limitante de que se restringe a una visión desde el lado de la oferta donde sólo importan las decisiones de localización de los trabajadores (cualificados). ${ }^{4}$ En este trabajo, adoptaremos este último enfoque (sectorial) para realizar una clasificación de sectores creativos a partir de los censos económicos y evaluar su efecto en la dinámica de crecimiento de las zonas metropolitanas del país.

\section{Presencia de las industrias creativas en las zonas metropolitanas}

\section{Definición y caracterización de las industrias creativas}

Este documento presenta una propuesta para medir a una fuerza creativa al interior de las ciudades a partir de la clasificación industrial de los censos económicos del Instituto Nacional de Estadística y Geografía (INEGI); en específico, se está interesado en evaluar un núcleo creativo de industrias que están más asociadas a las actividades científicas y técnicas. Al respecto, el sector 54 que los censos económicos reportan como Servicios Profesionales,

${ }^{4}$ En un libro reciente, Moretti (2012) también realiza una crítica a la visión de oferta (que está detrás de la noción de la clase creativa) en donde la prescripción de política pública va encaminada sólo a generar o incrementar las amenidades urbanas con el fin de revitalizar las economías de las ciudades. En particular, Moreti señala que hay que distinguir entre causa y efecto en el papel que tiene la clase creativa en ciudades exitosas como Seattle, Austin o Nueva York en Estados Unidos, ya que estas ciudades se volvieron atractivas porque fueron exitosas en construir una base económica sólida y no al revés. 
Científicos y Técnicos tiene, desde nuestro punto de vista, los atributos necesarios para identificar a este sector creativo duro. A su interior, existe heterogeneidad pues hay actividades (a nivel de rama, 4 dígitos) más orientadas a actividades científico-técnicas $(5413,5416,5417)$ y otras más orientadas al diseño y publicidad $(5414,5418) .{ }^{5}$ Sin embargo, el sector 54 puede verse como un sector que aglutina diversas actividades basadas en servicios profesionales (calificados) que están más orientadas a apoyar un sector industrial o de servicios creativo (diseño, desarrollo de software, consultoría especializada, entre otros). Para una justificación de esta clasificación véase también Fingleton y colaboradores (2007).

En relación con lo que la literatura ha discutido para clasificar a una clase creativa à la Florida (2002) —véase sección dos-, la propuesta que se hace a partir del sector 54 es ciertamente parcial pues sólo se estaría aproximando al conjunto del núcleo creativo de actividades científicas y técnicas, una parte importante del grupo de profesiones creativas (sobre todo aquellas actividades de consultoría admnistrativa y de abogados), e incluiría también algunas actividades asociadas al sector "bohemio" (artes gráficas, publicidad, arquitectura, etc.); pero el sector 54 no sería suficiente para contemplar al resto del sector "bohemio" como son las relacionadas con la creación artística (sub-sector 711), la edición (sub-sector 511) y la cinematografía (sub-sector 512), por citar algunas, como tampoco estaría incluyendo a otras ocupaciones profesionales creativas de consultoría asociadas a las finanzas y el sector inmobiliario (sector 52 y 53). De cualquier forma, creemos que el sector 54 - en general- es un excelente sector proxy de lo que son las industrias creativas en su conjunto.

${ }^{5}$ En específico, la clasificación de la clase técnica y creativa que emana del sector 54 de los censos económicos sería la siguiente. Clase creativa: Rama 5414 Diseño especializado, Rama 5418 Servicios de publicidad y actividades relacionadas, Subrama 54132 Servicios de arquitectura de paisaje y urbanismo, Subrama 54134 Servicios de dibujo, Subrama 54172 Servicios de investigación y desarrollo en ciencias sociales y humanidades, Subrama 54191 Servicios de investigación de mercados y encuestas de opinión pública, Subrama 54192 Servicios de fotografía, Subrama 54193 Servicios de traducción e interpretación. Clase técnica: Subrama 54133 Servicios de ingeniería, Subrama 54136 Servicios de levantamiento geofísico, Subrama 54138 Laboratorios de pruebas, Subrama 54162 Servicios de consultoría en medioambiente, Subrama 54169 Otros servicios de consultoría científica y técnica, Subrama 54171 Servicios de investigación y desarrollo en ciencias físicas, de la vida e ingeniería. 
Por otra parte, en la medición se propone poner atención también a otros sectores afines y/o cercanos al sector creativo, entre los que se encuentran: el sector 56 (servicios de apoyo a negocios), el sector 61 (servicios educativos), el sector 72 (hospedaje temporal y restaurantes) y, por supuesto, el sector 71 (servicios de esparcimiento cultural) que incluiría a otra porción del empleo bohemio. La inclusión de estos sectores se fundamenta además en la razón siguiente: si bien se busca identificar la presencia de un sector creativo, también interesa la presencia de un sector para atender las necesidades de estilo de vida y de consumo de ese sector creativo, que como se señaló, forma parte del atractivo para captar migrantes calificados. ${ }^{6}$

\section{Comportamiento económico reciente de las zonas metropolitanas de México}

Los datos más recientes del Censo de 2010 indican que la población de las 56 zonas metropolitanas fue de 62620980 personas, lo que implicó un crecimiento de $17.5 \%$ respecto del año 2000. Las zonas metropolitanas que crecieron más durante la década fueron las de Cancún (57\%), Puerto Vallarta (55\%), Reynosa (39\%), Pachuca (37\%), Querétaro (34\%), y Tuxtla Gutiérrez, Tijuana, Saltillo, Aguascalientes, Zacatecas, León y Villahermosa con un crecimiento cercano al 30\%. Todas estas ciudades comparten la característica de que fueron ciudades medias en 2000 (250 mil a un millón de habitantes) — salvo en el caso de León-, confirmando una tendencia ya presente desde la década de los noventa en donde precisamente este tipo de ciudades eran ya las que crecían a mayores tasas. Más adelante observaremos que algunas de estas ciudades medias también muestran un dinamismo importante de las clases de actividades asociadas al sector creativo.

${ }^{6} \mathrm{Al}$ respecto, Moretti (2012) sostiene que por cada ocupación creada en un sector de alta tecnología (en las zonas metropolitanas de Estados Unidos), se generan cinco empleos locales adicionales en los sectores de no alta tecnología en el largo plazo. Este efecto multiplicador de los trabajadores calificados opera principalmente en sectores locales de servicios (como restaurantes, servicios de salud, gimnasios, spas, peluquerías, etc.) que además no dependen de la competencia externa. 
En cuanto a la generación de Valor Agregado Censal Bruto (VACB), el conjunto de las 56 zM representó $73 \%$ y $67 \%$ del total del país, para los años 2003 y 2008 respectivamente (cuadro 1), lo que significó una caída de 5 puntos porcentuales en la participación de la actividad económica (en términos del VACB) de las zM dentro del contexto nacional. Por su parte, la participación de las zM en el empleo nacional aumentó ligeramente en el mismo periodo en 2 puntos porcentuales, al pasar de $73 \%$ en 2003 a $75 \%$ en 2008. En ese contexto, las industrias manufactureras (sector 31) siguen siendo, en el 2008, el sector con mayor participación en el empleo (23\%) y VACB (36\%) respecto del total de las zonas metropolitanas.

Sin embargo, se debe tener en cuenta que la participación del empleo manufacturero muestra una caída en su participación respecto de años anteriores (27\% en 2003), que se contrarresta por un incremento en la participación del empleo en los sectores asociados a servicios (38\% en 2003 y $45 \%$ en 2008).

Este crecimiento del sector terciario no es una tendencia nueva, sino que forma parte del proceso de terciarización de la economía mexicana en general y de las zM en particular, y que ya ha sido abordado en otros trabajos (Garza, 2006). El dato a resaltar del periodo 2003-2008 es que el sector manufacturero se mantuvo más competitivo que el sector servicios, al observar que su productividad creció a una tasa anual de $0.4 \%$, en contraste con un fuerte descenso en la productividad del conjunto de sectores de servicios con una tasa anual de $-7.5 \%$ (cuadro 1). Esta caída de la productividad en los sectores de servicios debe verse en el marco de un fuerte crecimiento de su empleo ( $8.4 \%$ anual), que ciertamente no produjo, al menos en el corto plazo, un incremento proporcional en el vacB.

\section{Presencia de las industrias creativas en las zonas metropolitanas}

Entre los sectores de servicios destacan por su participación en el total del empleo de las zm en 2008 (véase cuadro 1), el sector 56 de servicios de apoyo a negocios (8\%), el sector 61 de servicios educativos (3.5\%) y el sector 72 de alojamiento y restaurantes $(7.9 \%)$. Por lo que respecta al sector 54, representó 3.3\% del empleo total en las zM (no obstante que registró un ligero descenso en su participación respecto del año 2003 que fue de 


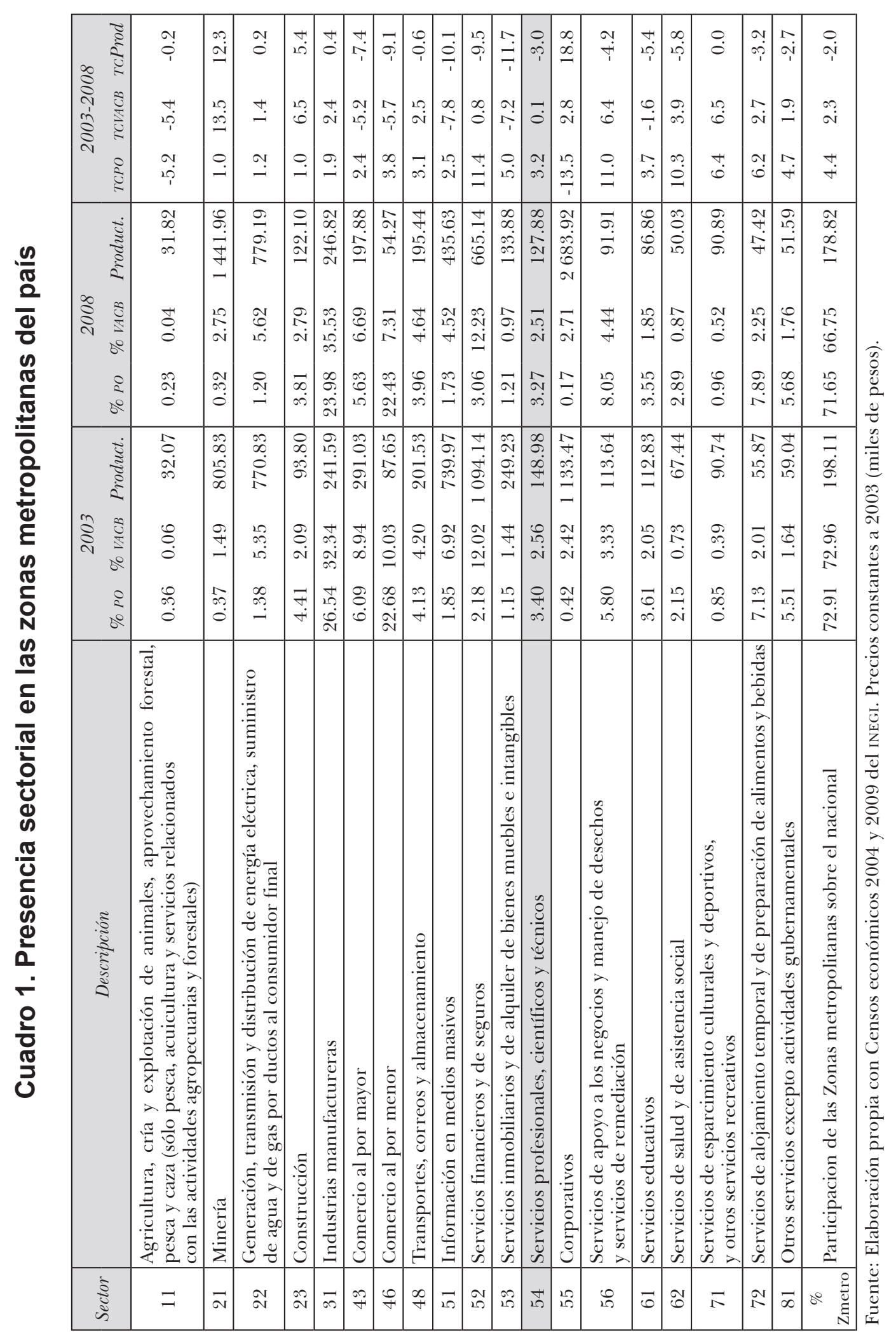


$3.4 \%$ ); y por lo que respecta al sector 71 (cultural), sólo representó $0.96 \%$ del empleo en $2008 .^{7}$

En general, el empleo en los sectores de servicios tuvo un fuerte incremento en el periodo 2003-2008 (el conjunto de servicios creció a una tasa anual de $8.4 \%$ ); y destaca el crecimiento en el sector 52 de servicios financieros con $11 \%$, el sector 56 con $11 \%$ y el sector 62 de servicios de salud con $10 \%$. En este contexto, el sector $54 \%$ creció sólo a una tasa anual de $3.2 \%$ en el periodo 2003-2008.

Como ya se señaló, el fuerte incremento del empleo en los sectores de servicios no generó un incremento proporcional en su vaCB, lo que afectó directamente en una fuerte caída de la productividad. En este contexto, los sectores que reportaron los mayores descensos fueron: el 53 (servicios inmobiliarios) con $-11.7 \%$ anual, el 51 (información de medios) con -10\% anual, y el 52 (servicios financieros) también con -10\% anual de caída de la productividad. Al respecto, debe señalarse que el sector 54 fue de las actividades económicas con menor caída en la productividad (-3\% anual), junto con el 72 de hoteles-restaurantes (-3.2\%) y el sector 71 (esparcimientos) que creció a una tasa cero. Es decir, los sectores asociados a la clase creativa

${ }^{7}$ En este momento vale hacer un apunte sobre el peso y la significancia del sector creativo en el total del empleo metropolitano nacional. Medido a partir del sector 54, el empleo creativo representó 3.3\% del empleo total en 2008. Si a este sector le sumamos el empleo del sector 71 (de esparcimiento cultural), el empleo del sector creativo ascendería a $4.3 \%$ aproximadamente. En un ejercicio de medición más detallado que se realizó a nivel de 3, 4 y 5 dígitos (Sistema de Clasificación Industrial de América del Norte, SCIAN) de clasificación de los censos económicos 2009, se identificaron a aquellas actividades que representaran de manera más precisa a las actividades creativas siguiendo los lineamientos de Florida (2012). Bajo esta clasificación se estimó que el sector creativo representaba $6.7 \%$ del empleo total que corresponde a un $3.7 \%$ del núcleo creativo, $1.5 \%$ del bohemio y $1.4 \%$ de profesiones creativas — dealer class- (Estos resultados pueden solicitarse al autor por correspondencia). Al comparar con estimaciones para otros países que analizan también industrias en vez de ocupaciones — como al adoptado en esta investigación-, encontramos mediciones semejantes; por ejemplo, Boix y Lazzeretti (2012) estiman una participación de las industrias creativas en España del 6.5\% (2007) respecto de la ocupación total, de Dinamarca 8.8\%, y de Alemania 7.2\% — por citar algunos países - ; por su parte, Méndez, Michelini, Prada y Tébar (2012) tienen estimaciones menores, calculando para España 3.8\% de participación, que según los autores, tiene un nivel medio respecto de los otros países de la Comunidad Europea, y en donde Finlandia $(6.2 \%)$ y Dinamarca $(5.7 \%)$ tendrían los niveles más altos de participación. 
tuvieron en general el "mejor desempeño" en términos de productividad en el periodo 2003 y 2008.

En cuanto al crecimiento del VACB, destaca en general el fuerte crecimiento promedio anual del sector 21 de minería (13.5\%) y el del sector 23 de construcción (6.5\%); en los sectores de servicios, destaca el sector 71 de esparcimientos culturales (6.5\%), y el sector 56 de servicios de apoyo a negocios (6.4\%). En este sentido, el sector 54 sólo creció su VACB a una tasa anual de $0.1 \%$, muy cercano al $0.2 \%$ del crecimiento agregado de los sectores asociados a servicios.

Para tener una primera aproximación regional del sector creativo, se presenta la participación del empleo del sector 54 en cada zM utilizando un gráfico radial ordenado de menor a mayor participación (véase figura 1). El dato nacional es de $2.84 \%$ (véase círculo en la serie de la figura 1 ) y en el conjunto de las zM es de $3.3 \%$. Pero entre las zonas metropolitanas, la participación del empleo del sector 54 al interior de cada zM es heterogénea. Si analizamos el gráfico conforme las manecillas del reloj, observaremos que en los primeros 180 grados se encuentran las zM con menor participación y en donde apenas se alcanza hasta un $2.5 \%$ de participación. Nótese que el dato nacional se ubica casi exactamente al inicio del último cuarto cuadrante de la serie, y a partir de ahí se localizan las ZM con mayor participación del sector 54: Villahermosa (4.2\%), zм Ciudad de México (4.2\%), Córdoba (3.9\%), Querétaro (3.8\%), Mérida (3.7\%), Monterrey $(3.6 \%)$, Tepic $(3.5 \%)$, Jalapa (3.2\%), Cancún (3\%), Morelia (3\%), Zacatecas $(2.9 \%)$, Tampico (2.9) y Guadalajara (2.9\%).

El cuadro 2 muestra diferentes indicadores de desempeño del sector 54 en las ZM durante el periodo 2003-2008 (productividad, empleo y VACB). En general, puede concluirse que el sector 54 tiene su mejor desempeño en la zm Valle de México, Querétaro, Cancún, Villahermosa, Monterrey, entre otras, que son una combinación entre zonas metropolitanas grandes y medias.

\section{Concentración del empleo y centralidad metropolitana}

En la sección anterior se mostró que las actividades asociadas a los sectores creativos - en particular el sector 54- mostraron, en general, a pesar del 
Figura 1. Gráfico radial de la participación del sector 54 en el total de la población ocupada de las zm (2008)

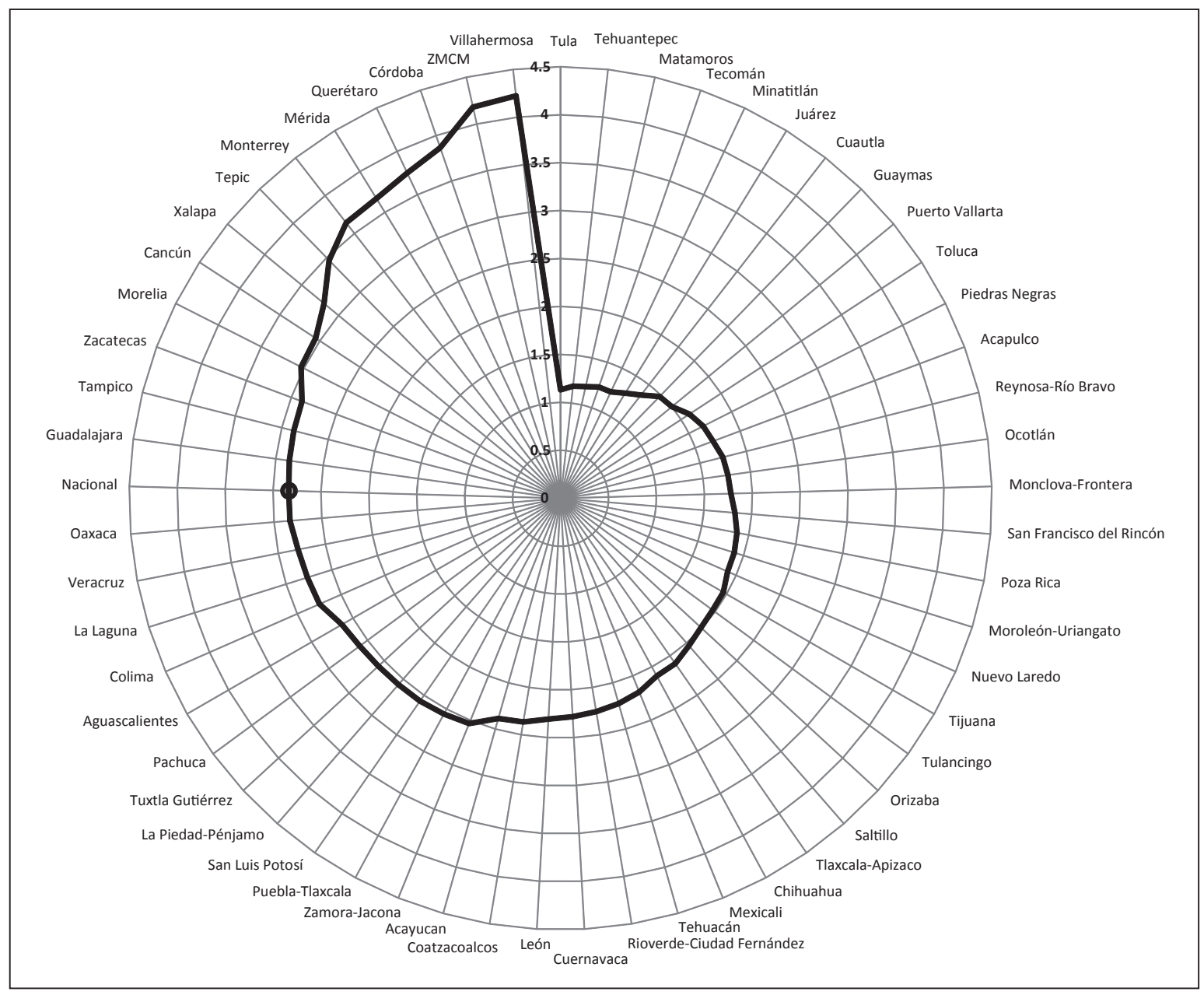

Fuente: Elaboración propia con datos de los Censos Económicos 2009 del INEGI.

clima económicamente adverso que empezó a vivir el país a partir de la crisis económica mundial de 2008, un desempeño económico aceptable en los años de estudio de esta investigación (2003-2008).

Ahora el objetivo en esta sección es evaluar si la actividad económica relacionada con la clase e industria creativa, presenta algún patrón característico en la concentración espacial de su empleo. Como se discutió anteriormente, uno de los rasgos característicos de las actividades económicas relacionadas con la clase creativa es su ubicación próxima al centro 


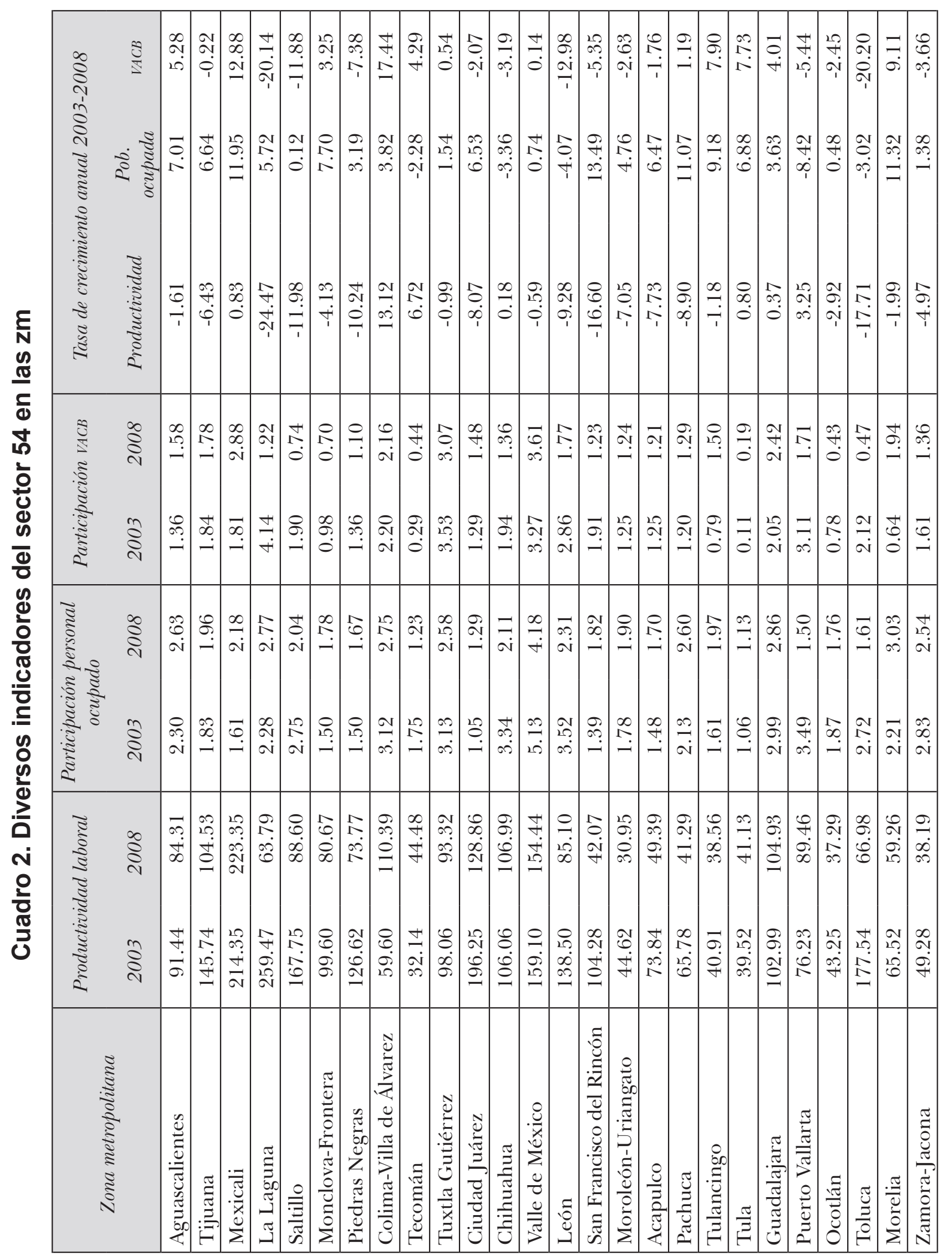




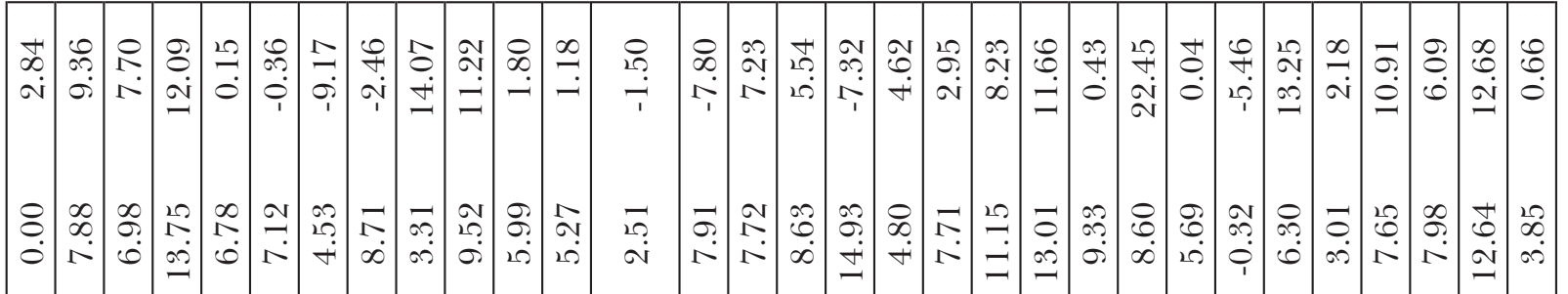

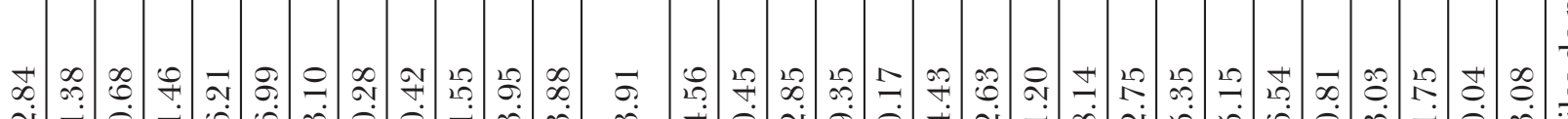

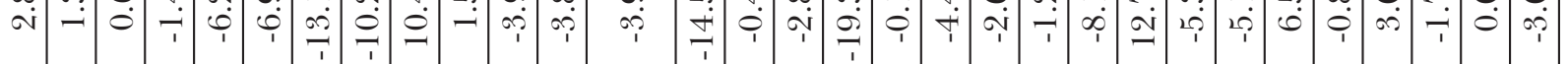

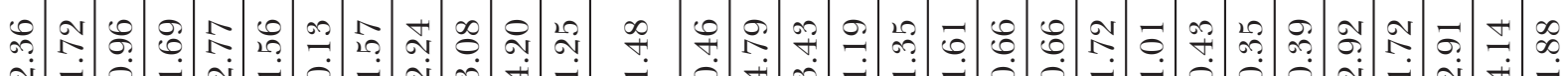

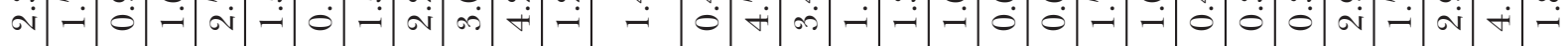

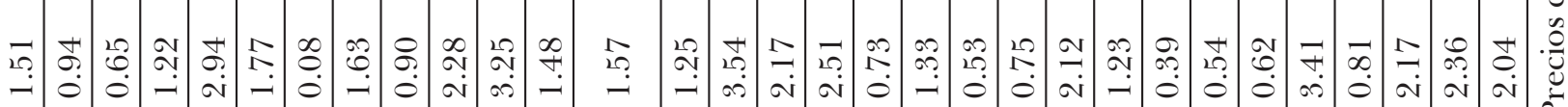

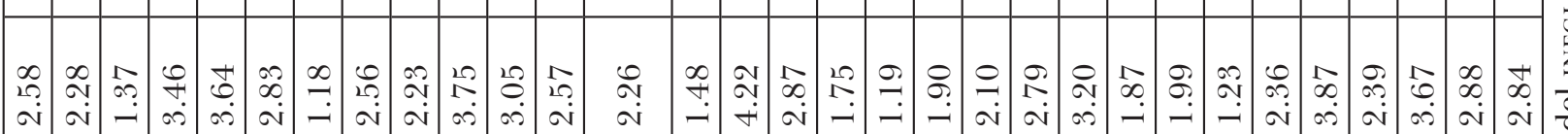

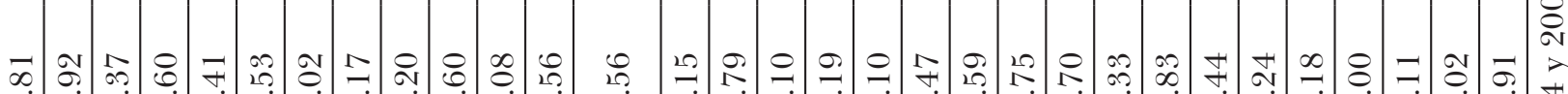

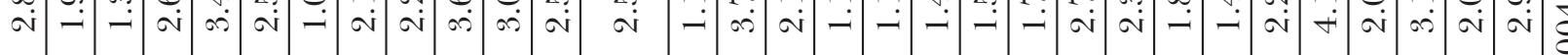

б.

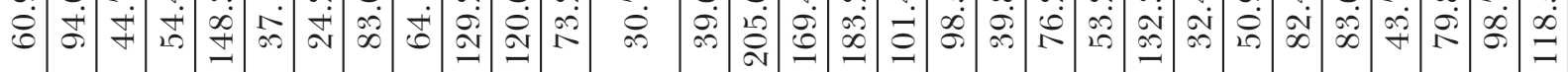

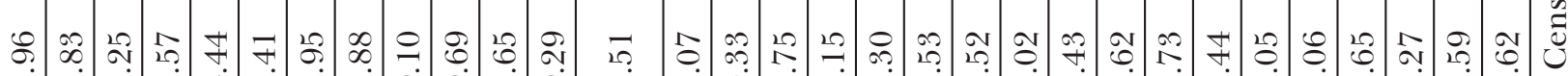

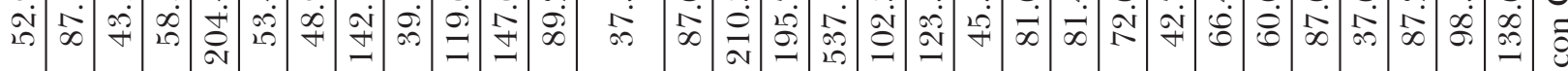

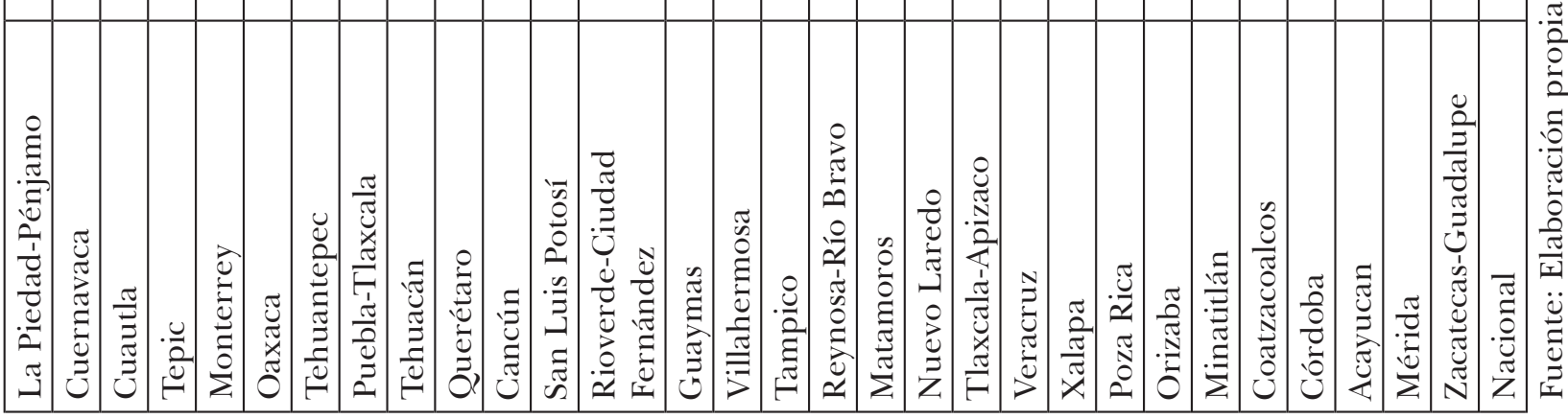


económico de las ciudades, ya que con ello se potencia la generación de externalidades positivas que se asocian de manera intrínseca a estos sectores creativos.

Para analizar la concentración del empleo y de la población en las 56 zonas metropolitanas del país, recurrimos a una escala de AGEB. El primer paso fue designar (y localizar) ad hoc al zócalo o plaza central de cada una de las 56 zM como el "centro económico". Una vez identificado este punto geográfico, se examinó la densidad del empleo en cada AGEB perteneciente a una zM respecto del centro económico identificado. ${ }^{8}$

Según los resultados obtenidos con la medición hecha en este trabajo, y tomando como base la información de los censos económicos 2008, se obtuvo que: $46 \%$ del empleo total de las zonas metropolitanas se concentra en un radio de 5 kilómetros a partir de los zócalos o plazas centrales, a $10 \mathrm{kms}$ se concentra $72 \%$, a $15 \mathrm{kms} 86 \%$, y a $20 \mathrm{kms} 93 \%$. En cuanto a la población $2010,31 \%$ se concentra en un radio de $5 \mathrm{kms}$ desde las plazas centrales, a $10 \mathrm{kms}$ se concentra $57 \%$, a $15 \mathrm{kms} 75 \%$ y a $20 \mathrm{kms} 86 \%$. Para ilustrar los porcentajes mencionados, en la figura 2 se muestran las curvas de acumulación de empleo y de población por cada kilómetro de alejamiento a partir de los zócalos o plazas centrales de las zonas metropolitanas.

La figura 2 muestra que en el agregado de las zM, la gente tiende a desplazarse más hacia el centro de la ciudad por motivos de trabajo pero vive menos cerca de éste. Sin embargo, este diferencial no oculta el hecho de que prevalece una fuerte correlación entre localización del empleo y localización de la vivienda a nivel de AGEB a lo largo de las zM. Los resultados aquí obtenidos son coincidentes con los observados en las zonas metropolitanas de Estados Unidos en un estudio realizado por Glaeser y Kahn (2001), a nivel de código postal, señalando que 25\% del empleo se

${ }^{8}$ Se analizaron 22421 AgEBs de las ZM a partir de las cartografías urbanas generadas por el INEGI para el año 2005. La información de empleo a nivel de AGEB proviene de los censos económicos 2009 y también fueron proporcionados — para fines de investigación- por el INEGI. La información proporcionada por esta dependencia fue posible gracias al convenio número 11557-053-11-1-02 suscrito entre el INEGI y la UNAM el 15 de octubre de 2002 y bajo el cual el Centro Regional de Investigaciones Multidisciplinarias de esta universidad solicitó información mediante el documento núm. de folio CRIM/D/010/2008. 
Figura 2. Curvas acumulativas de la población y del personal ocupado respecto del centro económico de las ZM

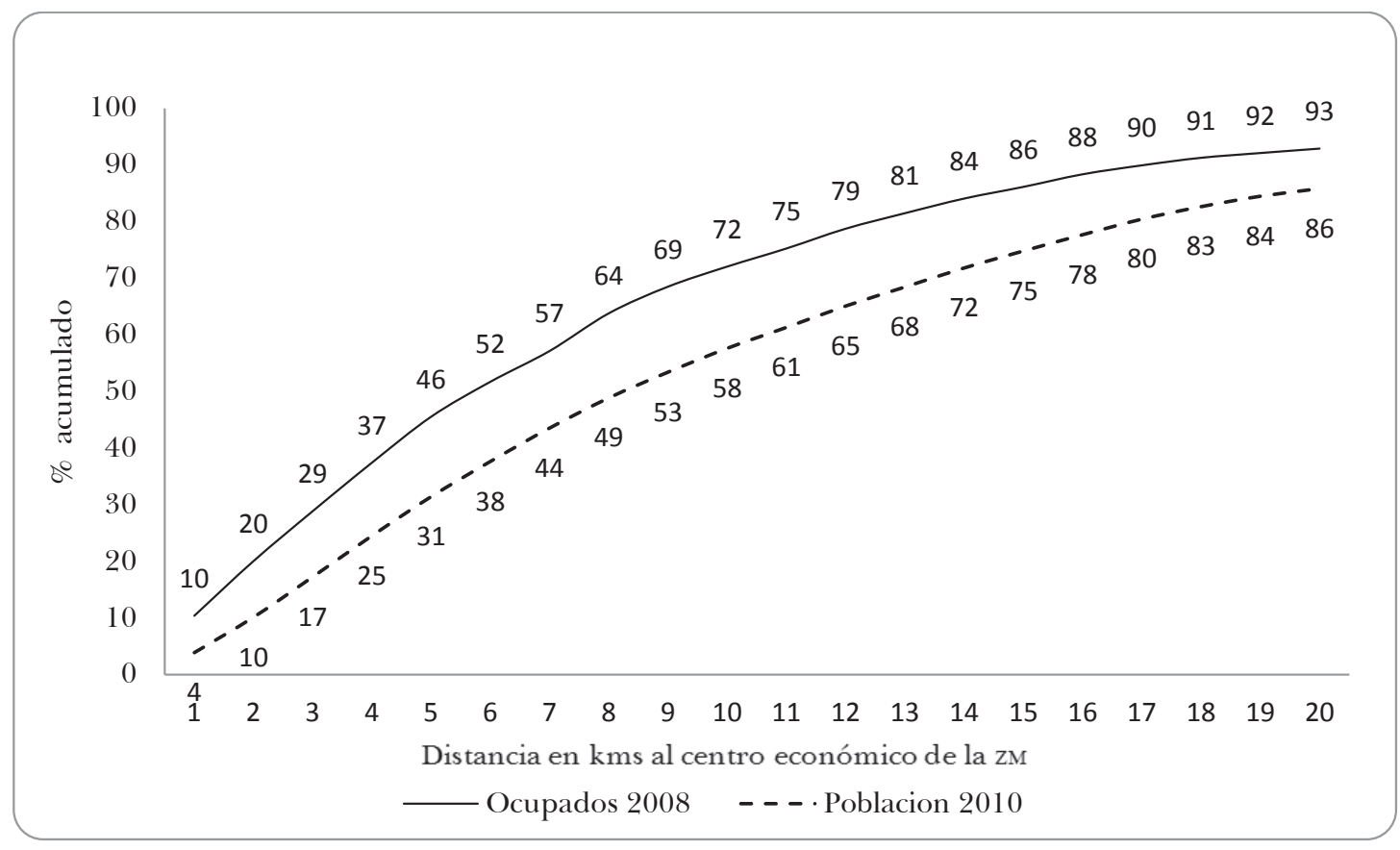

concentra dentro de los primeros $5 \mathrm{kms}$ respecto de los distritos centrales financieros de las ciudades (Central Business District, CBD). ${ }^{9}$

Por su parte, las curvas de acumulación del empleo desde el centro económico por sector de actividad (manufactura, comercio y servicios), se muestran en la figura 3 -se incluye también la serie del empleo en el subsector 541 y se muestran los valores puntuales de las series, a excepción del comercio- . En primera instancia, se destaca la tendencia de la curva de concentración del empleo manufacturero, por mantenerse debajo de las curvas de servicios y comercio, pero llama la atención su repunte cuando la distancia lineal al centro es $12 \mathrm{kms}$ y se ubica ligeramente por arriba de la curva del empleo comercial. La curva acumulada de servicios en general siempre tiende a estar por arriba que la manufactura y el comercio,

${ }^{9}$ Fue Burguess —representante de la corriente de pensamiento de ecología urbana de la Escuela de Chicago- quien propone, en su modelo de estructura urbana de anillos concéntricos, la designación del centro de las ciudades como Central Business District (CBD) o distrito central de negocios o financiero (Richardson, 1993). 
Figura 3. Curvas acumulativas de empleo respecto del centro económico por gran sector de actividad económica en las $56 \mathrm{zm}$ (2008).

Se incluye la serie de empleo del subsector 541

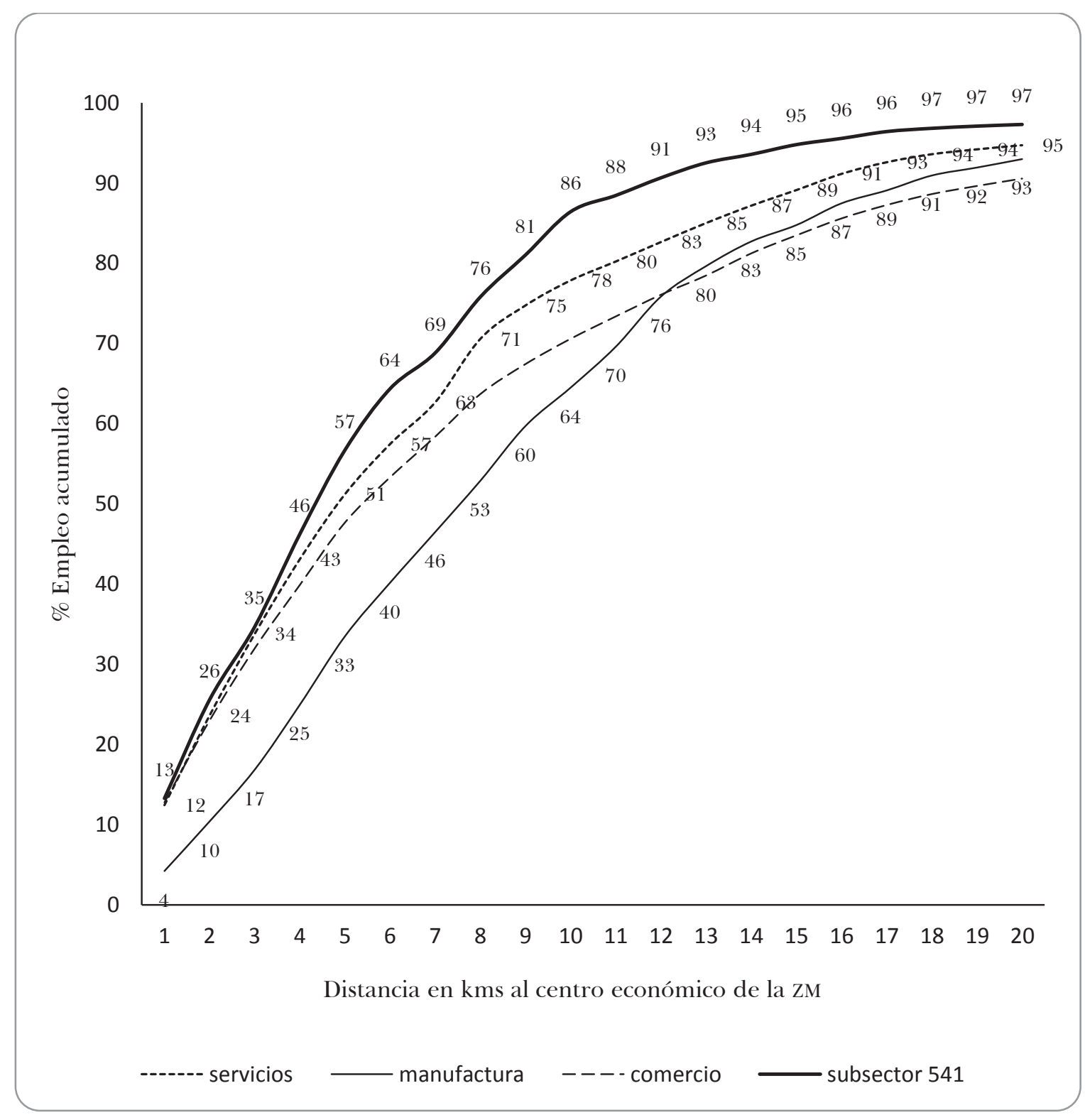


salvo en los primeros 3 kilómetros en los que comparte similares niveles de concentración de empleo que el sector comercio. En general, los datos de la figura 3 a nivel de gran sector indican que el empleo manufacturero en promedio está más desconcentrado en las zm del país, y en contraste el empleo en el sector servicios tiende a estar más concentrado. En particular, dentro de un radio de $5 \mathrm{kms}$ (que concentra $46 \%$ del empleo total), el sector servicios concentra $51 \%$ del empleo, el sector manufacturero $34 \%$ y el sector comercio $48 \%$.

Interesante por destacar en la figura 3 es el comportamiento del subsector 541 que siempre se encuentra por arriba de todas las curvas. Si bien dentro de los primeros $3 \mathrm{kms}$ no hay una diferencia importante en la acumulación de empleo entre el subsector 541 y el total de empleo de servicios, en los $5 \mathrm{kms}$ se observa ya una diferencia de 6 puntos porcentuales a favor del subsector 541 que se mantiene relativamente constante hasta el final de la curva. Este comportamiento sugiere una mayor concentración del empleo en el subsector creativo en relación con el resto de las actividades económicas (véase más adelante).

Si bien el porcentaje del empleo dentro de un radio de distancia desde el centro de la ciudad es un indicador de la concentración de empleo en una ciudad, no es el único y puede suscitar algunas reservas. En particular, esta medición se ve afectada por el tamaño-extensión de la ciudad; por ejemplo, ciudades grandes como la zMCM tendrán de manera "natural" una menor concentración del empleo. En este sentido, algunos autores han propuesto que la densidad de empleo respecto del centro económico debería medirse bajo el supuesto de "una distribución exponencial"

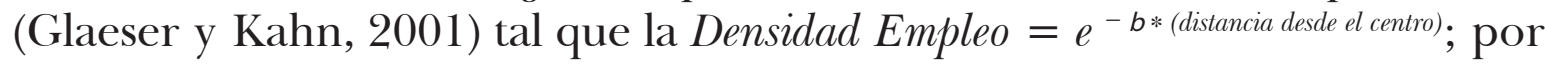
lo tanto, al tomar, el logaritmo natural (Ln) de ambos lados de la ecuación, la expresión se transforma en Ln Densidad $=-b *$ Distancia. Este supuesto ha derivado en considerar la regresión lineal de esta relación como una medición de la concentración del empleo o gradiente del empleo

$$
\operatorname{Ln}\left(\frac{\text { Empleo }}{K m^{2}}\right)=a_{Z M S}-b * \text { Distancia }+e
$$

Donde alfa es una constante, beta es el parámetro asociado a la distancia y epsilon es una perturbación estocástica. 
A continuación se presentan los resultados de la estimación de la regresión de gradiente de empleo (ecuación 1) para las 56 zonas metropolitanas del país. En el ejercicio se consideraron 22441 AGEBs y se transformó la variable dependiente en $\mathrm{Ln}\left(1+\right.$ Empleo $\left./ \mathrm{Km}^{2}\right)$ con la finalidad de involucrar en el análisis aquellos AGEBs con cero empleo (se encontraron 1449 AGEBs bajo esta condición); $b<0$ evalúa la hipótesis de que la cercanía al centro económico condiciona la densidad del empleo a nivel AGEB y es conocida en la literatura como el gradiente de empleo que indica el nivel de concentración del empleo respecto del centro de las ciudades. Los resultados de la regresión por mínimos cuadrados ordinarios (MCO) estiman un coeficiente beta de -0.036 y estadísticamente significativo con $99 \%$ de confianza.

Por su parte, Glaeser y Kahn (2001) hallaron para el caso de las ciudades de Estados Unidos un coeficiente de -0.169 con un error estándar de 0.002; es decir, comparativamente, las ZM de México son mucho más descentralizadas. Resulta interesante la estimación cuando se elimina la constante o el efecto fijo promedio de las zM ya que el coeficiente de la densidad de empleo se vuelve positivo 0.24, debido a que la densidad a nivel AGEB está positivamente correlacionada con el tamaño-extensión de la ciudad (lo mismo ocurre en Estados Unidos pero no con la intensidad de las zM de México).

Para analizar el nivel de concentración del empleo en el conjunto metropolitano del país, se estima la ecuación del tipo 1 por separado para cada una de las ZM a escala de AGEB. La distribución del gradiente de empleo se puede observar en la figura 4 . Un primer dato a destacar es que prevalece una fuerte heterogeneidad en la estimación del gradiente de empleo a lo largo de las zonas metropolitanas. Si se considera a las zM más grandes del país (zмcm, Guadalajara, Monterrey y Puebla), Guadalajara destaca como la urbe con mayor grado de concentración con una beta de -0.18 , mientras que el resto de las zm registran un gradiente muy parecido: -0.10 para Monterrey, -0.079 para la zMCM y -0.075 para la zM de PueblaTlaxcala. En general, las zonas metropolitanas de la región del centrooccidente y centro norte del país obtuvieron niveles de concentración del empleo elevados: zm Río Verde -0.64, zm Zamora -0.38, zM SLP -0.35, zM de Aguascalientes -0.29 y zM de Morelia (-0.22). Asimismo, en esta región se tienen dos observaciones extremas, San Francisco del Rincón y MoroleónUnriangato con $b<-1$. 
Figura 4. Distribución del gradiente de empleo en las ZM

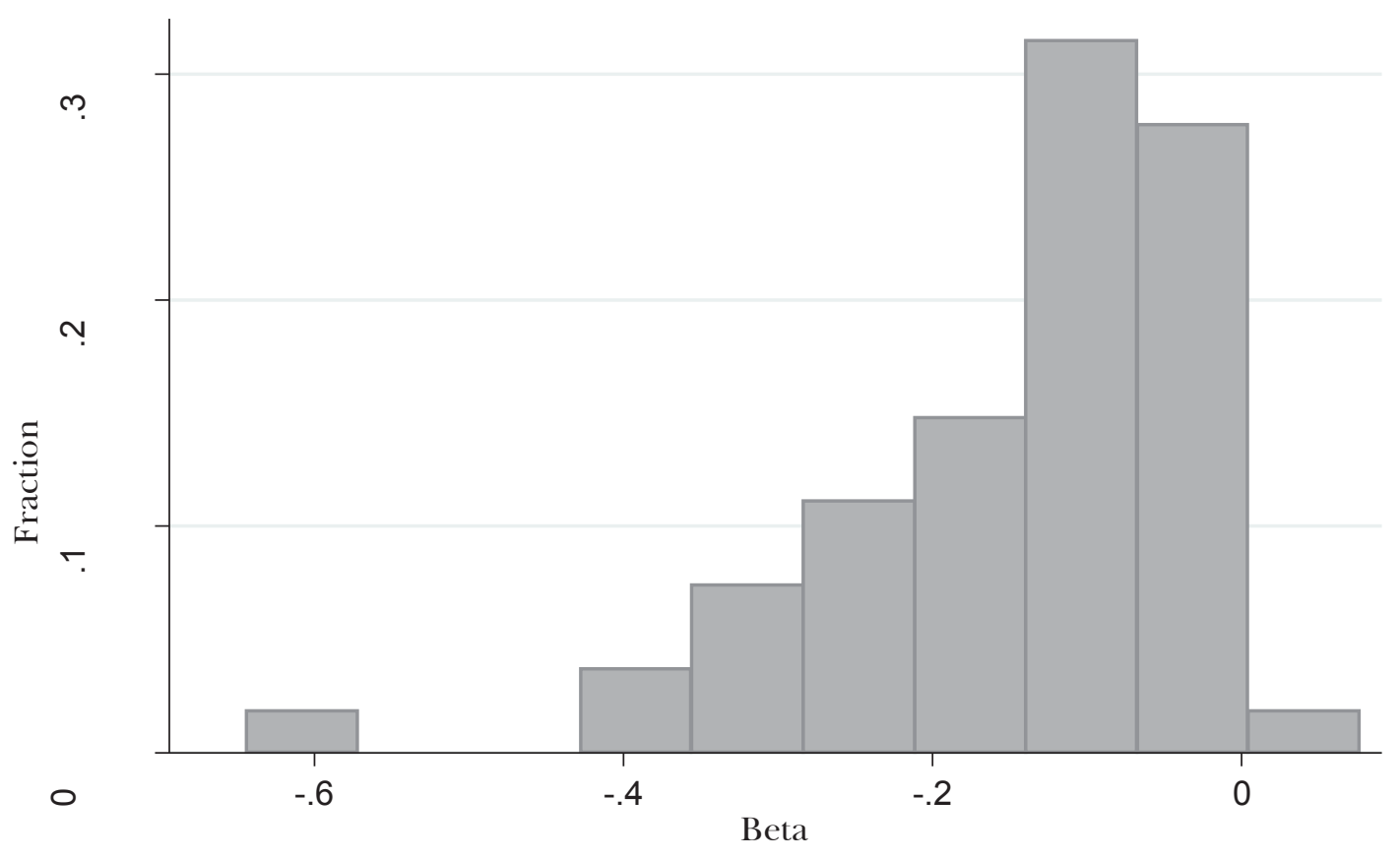

No se consideraron las zms con beta menor a -1 (Moroleón-Uriangato y San Fco. del Rincón).

Otras zm del centro del país y de la región golfo-sureste del país como Toluca, Querétaro, Léon, Minatitlán, Villahermosa, Córdoba y TlaxcalaApizaco, presentaron niveles "bajos" de concentración del empleo parecidos a los de la ZMCM; pero también hay otro grupo de zM en esta región del centro-sur que registra niveles altos de concentración del empleo: Cuernavaca -0.22 , Oaxaca -0.20 , Orizaba -0.17 , Pachuca -0.17 y Coatzacoalcos -0.17. Cabe señalar que en las estimaciones sobresalen Tula, Veracruz y Tehuantepec como las zM con el menor nivel de concentración de empleo del país.

Las regresiones de empleo también se realizaron a nivel de los 68 subsectores (tres dígitos del SCIAN) que conforman los censos económicos; en el cuadro 3 se muestran los resultados obtenidos y son presentados en orden ascendente, es decir, de mayor a menor concentración espacial del empleo respecto del centro económico metropolitano. Destaca que los tres subsectores con mayor concentración son el 722 (restaurantes), 541 (servicios profesionales, técnicos y científicos) y 561 (servicios de apoyo a 


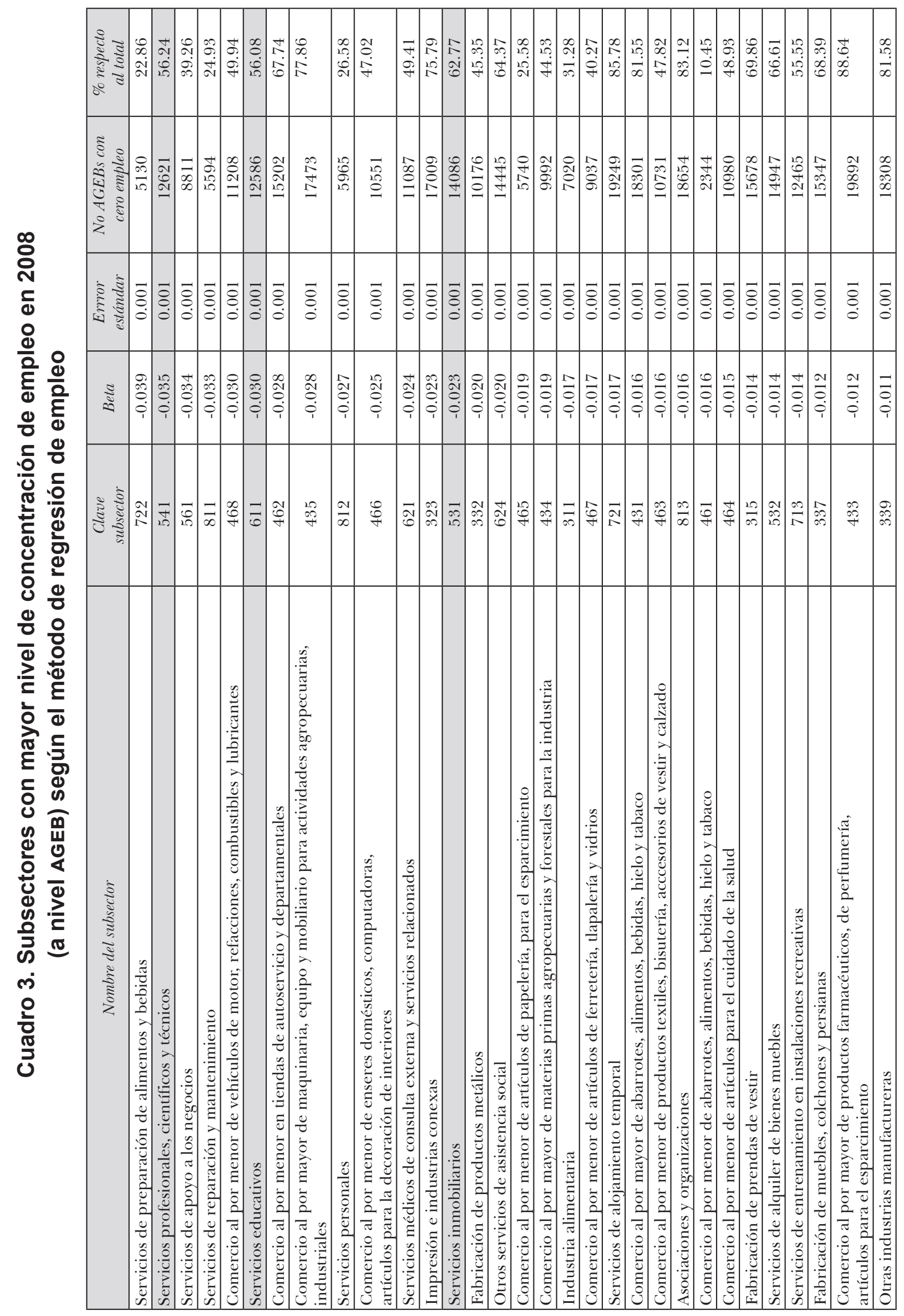


光 की

ஃ

คิ

官

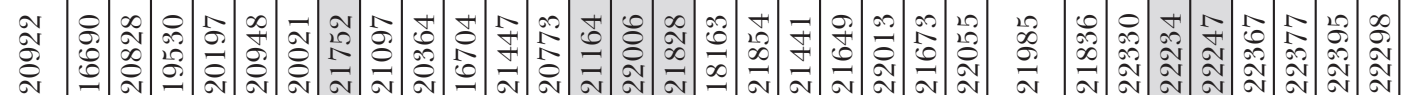

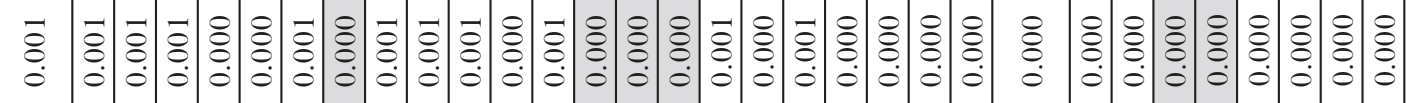

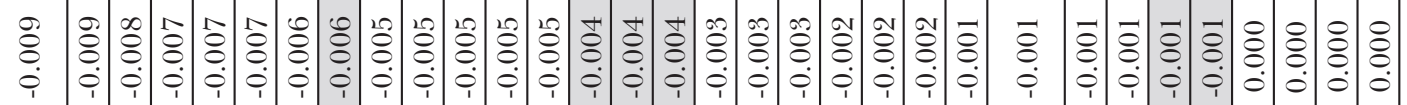

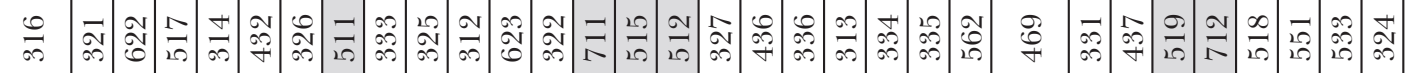

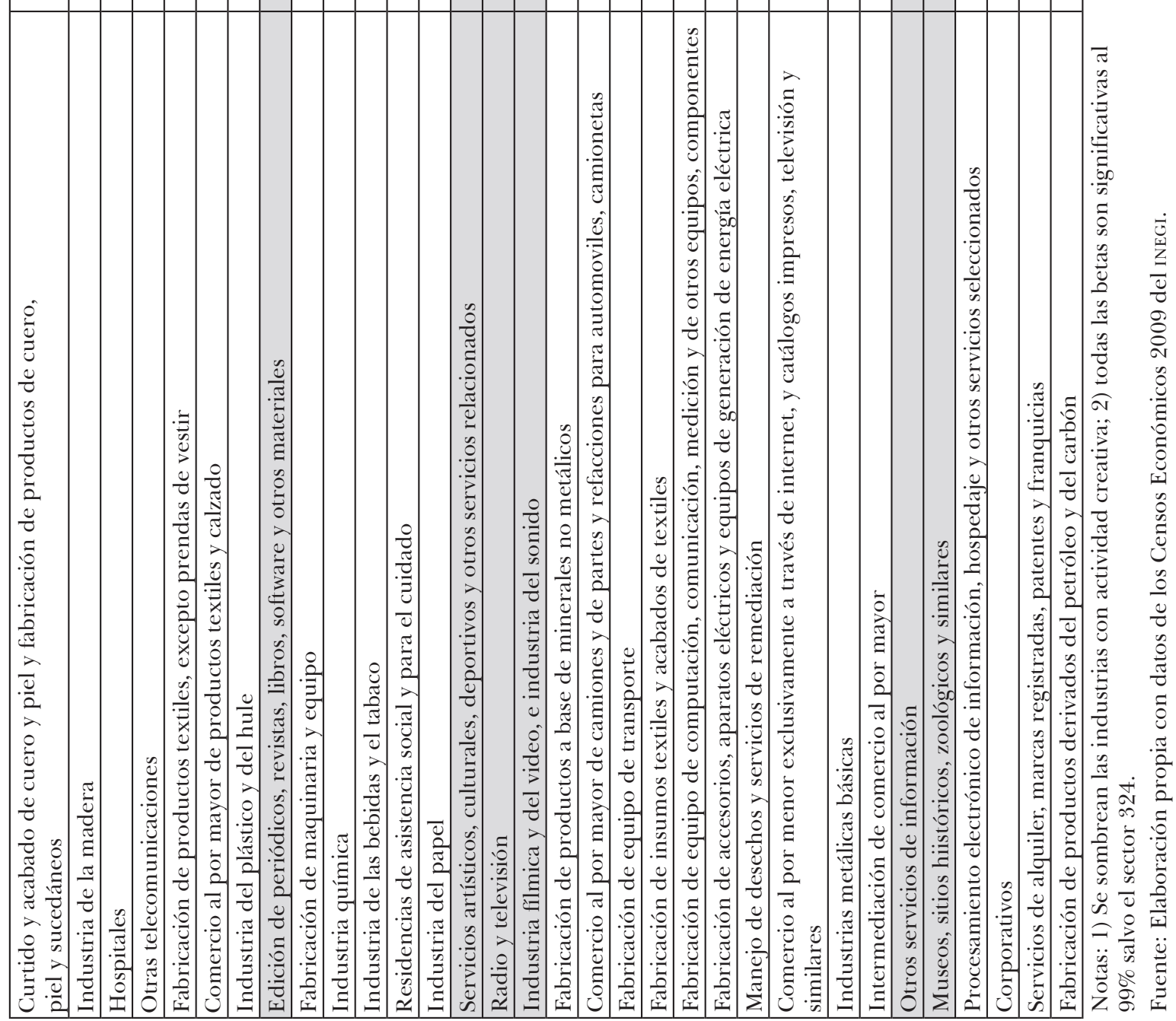


los negocios). En el sexto lugar también aparecen los servicios educativos (611) como uno de los más concentrados. Sin embargo, los subsectores asociados a la cultura y el esparcimiento $(711$ y 712$)$ y a la difusión y edición de medios $(511,512,515$ y 519) se localizan en la parte baja de la tabla mostrando bajos niveles de concentración espacial. Es decir, se corrobora que aquellas actividades asociadas a la clase creativa - tal como la teoría lo esperaría- despliegan un patrón de fuerte concentración espacial del empleo respecto de la distancia que guardan con el centro económico de las ZM; pero sólo se observa en aquellas actividades asociadas más al núcleo creativo y a las profesiones creativas (en particular, el subsector 541) ya que las industrias asociadas a las actividades bohemias muestran un fuerte nivel de descentralización.

\section{Efecto de la industria creativa en el crecimiento económico}

En esta sección tenemos una primera aproximación a si la clase creativa asociada al sector 54 ha tenido algún efecto en el crecimiento económico de las zM. Para tal propósito ahora se analiza la información agregada para cada ZM para los años 2003 y 2008 — que corresponden a los años de los censos económicos-, y se opta por estudiar un modelo estándar de convergencia de sección cruzada, de la productividad laboral de las zM para el periodo correspondiente 2003-2008 atendiendo a la siguiente especificación econométrica:

$$
g_{i, T}=b y_{i, 0}+a X_{i, 0}+m+e_{i}
$$

Donde $\left(g_{i, T}\right)$ es el crecimiento promedio para el periodo de análisis (2003-2008) del Valor Agregado Censal Bruto por persona ocupada (productividad laboral) de la Zona Metropolitana $i$ medido como $\ln \left(y_{i, r} / y_{i, 0}\right)$ con el logaritmo de la productividad laboral en el año inicial $y_{i, 0}$, otras variables relevantes $(X)$ para las $i$ Zonas Metropolitanas en el año inicial, una constante $m$ y epsilon como una perturbación estocástica. ${ }^{10}$

${ }^{10}$ Como es ampliamente conocido en la literatura de crecimiento, la ecuación (2) puede justificarse bajo un modelo neoclásico à la Barro (Barro y Sala-i-Martin, 1992) o simplemente como parte de una estrategia empírica para analizar procesos 
En el modelo 1 del cuadro 4 se muestran los resultados del modelo de convergencia absoluta (i.e. $a \neq 0$ ) estimado a partir de MCO, para las zonas metropolitanas del país. Los resultados de este primer modelo indican que no hay evidencia de convergencia en el crecimiento de la productividad laboral: la beta asociada a la productividad del año inicial 2003 es estadísticamente no diferente de cero. Este resultado es consistente con lo que múltiples estudios - bajo diferentes enfoques - sobre convergencia regional han llevado a cabo en México a nivel estatal —en vez de zona metropolitana-, y que muestran en primera instancia la ausencia de convergencia absoluta que es detectado a partir del proceso de liberalización económica de mediados de los ochenta (Esquivel, 1999; Calderón y Tykhonenko, 2007; Asuad y Quintana, 2010).

Este resultado sugiere que la convergencia puede estar condicionada por las diferencias regionales que son intrínsecas a cada zona metropolitana del país; por ejemplo, la dotación de capital humano varía indudablemente a lo largo de las zm y éste podría ser un factor que podría estar condicionando la convergencia de la productividad laboral.

En el cuadro 4 se propone un modelo de convergencia condicional que atiende a algunos de los componentes asociados al papel de la clase creativa en el crecimiento de las ciudades; al respecto, se propone que algunas variables del vector $\mathrm{X}$ de la ecuación (2) podrían estar condicionando la dinámica de convergencia. De manera específica, en el modelo se propone que los años de escolaridad promedio de la población

de catching up en el largo plazo (Baumol, 1986). Cuando en la ecuación de crecimiento $a=0$, se tiene la forma funcional para evaluar convergencia absoluta de la productividad, la cual se verifica cuando se estima econométricamente un parámetro $b<0$ y estadísticamente significativo. Bajo este caso se dice que las zonas metropolitanas estarían alcanzando el mismo valor estacionario de crecimiento. De igual forma, cuando $a \neq 0$ en la ecuación (2), se tiene la ecuación para evaluar convergencia condicional de la productividad, y esto se corrobora cuando además de estimar un alfa diferente de cero se obtenga también un $b<0$ y estadísticamente significativo. La implicación de crecimiento regional en este último caso indica que el vector de variables $X$ de la ecuación (2) captaría la heterogeneidad entre las zonas metropolitanas debida a la diferencias de las condiciones iniciales (en variables tales como el capital humano o la clase creativa) en el proceso de crecimiento metropolitano; cada zona metropolitana alcanzaría su propio estado estacionario debido a esta heterogeneidad en las condiciones iniciales. 


\section{Cuadro 4. Modelos de convergencia absoluta y condicional de sección cruzada de la productividad laboral en las zm, 2003-2008}

\begin{tabular}{|lccccc|}
\hline \multicolumn{1}{|c}{ Variables } & $\begin{array}{c}\text { Modelo 1 } \\
\text { Coef. }\end{array}$ & $\begin{array}{c}\text { Modelo 2 } \\
\text { Coef. }\end{array}$ & $\begin{array}{c}\text { Modelo 3 } \\
\text { Coef. }\end{array}$ & $\begin{array}{c}\text { Modelo 4 } \\
\text { Coef. }\end{array}$ & $\begin{array}{c}\text { Modelo 5 } \\
\text { Coef. }\end{array}$ \\
\hline Constante & 0.614 & -0.125 & -0.072 & -0.148 & -0.064 \\
& $(0.621)$ & $(0.474)$ & $(0.496)$ & $(0.515)$ & $(0.572)$ \\
\hline Log Pr 2003 & -0.156 & $-0.269^{*}$ & -0.219 & -0.150 & $-0.271^{*}$ \\
& $(0.128)$ & $(0.132)$ & $(0.133)$ & $(0.207)$ & $(0.135)$ \\
\hline Escol. prom. & & 0.081 & $0.125^{*}$ & $0.121^{*}$ & 0.085 \\
2005 & $(0.057)$ & $(0.057)$ & $(0.059)$ & $(0.056)$ \\
\hline Saldo neto & & $-0.006^{*}$ & $-0.005^{*}$ & $-0.005^{*}$ & $-0.006^{*}$ \\
migratorio & $(0.002)$ & $(0.002)$ & $(0.002)$ & $(0.002)$ \\
\hline Log Pr sector & $0.147^{* *}$ & & & 0.159 \\
54, 2003 & $(0.080)$ & & & $(0.097)$ \\
\hline Log Pr sector & & & $-0.045^{*}$ & \\
31, 2003 & & & $(0.101)$ & \\
\hline Log Pr sector & & & & 0.004 \\
56, 2003 & & & & $(0.058)$ \\
\hline Log Pr sector & & & & \\
61 2003 & & & & & \\
\hline
\end{tabular}

Notas: Los errores en paréntesis. * signifcancia de 1\%; ** significancia de $5 \%$. Se estimó bajo mínimos cuadrados ordinarios y los errores son robustos.

Fuente: Elaboración propia con datos de los Censos Económicos 2004 y 2009 del INEGI.

en 2005 (proxy de capital humano), el saldo migratorio neto en el año 2000 y el logaritmo de la productividad laboral de la clase 54 en el año 2003, condicionan la dinámica de convergencia regional entre las zM del país.

Las estimaciones indican que bajo esta especificación se genera convergencia en la productividad laboral entre las zM (véase que el coeficiente asociado a la productividad inicial es negativo y estadísticamente significativo), y además la productividad de la clase 54 tiene un efecto positivo significativo sobre el crecimiento de la productividad, mientras que el saldo 
migratorio neto lo hace de manera negativa (con un nivel significativo de al menos $10 \%) .{ }^{11}$

Los resultados del modelo 2 del cuadro 4 sugieren que hay una relación importante entre el saldo migratorio neto del 2000 y la productividad laboral del sector 54 en el año 2003. En específico, un efecto negativo del saldo migratorio neto en la ZM contribuye favorablemente en la convergencia pero sólo en el contexto de que prevalezca un dinamismo en sectores asociados a las actividades de la clase creativa. Para sostener este punto, véanse los resultados del modelo 3 del cuadro 4 que elimina la variable de productividad de la clase 54: el saldo migratorio continúa siendo significativo y ahora el capital humano se vuelve significativo, pero el nivel de significancia del coeficiente beta disminuye e incluso ya no es estadísticamente significativo al $10 \%$.

Es decir, el stock de capital humano no es suficiente para garantizar convergencia en presencia de un saldo neto migratorio negativo en la ZM, ya que se requiere además cierto tipo de actividad calificada que dinamice el crecimiento de las ciudades. ${ }^{12} \mathrm{Al}$ respecto, en el modelo 4 del cuadro 4 se presenta una especificación donde se añade la productividad laboral del sector 31 (industrias manufactureras). Destaca cómo el parámetro de convergencia pierde significancia, lo que indica que es cero. Finalmente, en el modelo 5 del cuadro 4 se propone una especificación donde se elimina la productividad del sector 31 pero se añade la productividad de las clases

${ }^{11}$ El hecho de que hay un efecto negativo del saldo migratorio sobre la convergencia no debe extrañar, ya que es inmediato pensar que en las zonas metropolitanas "maduras" (por ejemplo la zмсм y Guadalajara) crecerá su productividad a menor ritmo, lo que provocará una mayor salida de su población. Efectos negativos de la tasa neta de migración en modelos de convergencia como el aplicado aquí pueden encontrarse en Kirdar y Saracoglu (2008).

${ }^{12}$ En este sentido, estos resultados reforzarían la idea discutida en la sección 2 de que la clase creativa no puede ser reducida sólo a una expresión del capital humano. Mendoza (2013), en un trabajo que analiza convergencia condicional a nivel de ciudades en México para el periodo 1990-2005, encuentra convergencia condicional con efecto positivo de capital humano, pero en su modelo no involucra el saldo migratorio; sin embargo, en su estudio también estima un modelo de convergencia condicional espacial con externalidades espaciales significativas del PIB per cápita de las ciudades próximas, lo que sugiere que otras variables — asociadas al capital humano- también podrían estar condicionando la convergencia entre ciudades (como podrían ser la clase creativa y/o la movilidad intermetropolitana). 
asociadas a las clase creativa $(54,56$ y 61$)$. Los resultados indican que hay convergencia y que sólo el sector 54 tiene un efecto significativo (y positivo) sobre dicho proceso.

\section{Externalidades en la creación de empleo en los sectores asociados al sector creativo}

Uno de los efectos que se espera produzcan las actividades relacionadas con las industrias creativas, es la generación de derramas (spillovers) o externalidades; en particular, estas actividades económicas deben ser capaces de desarrollar derramas en la creación de empleo. ${ }^{13}$ Replicando un ejercicio propuesto por Fingleton y colaboradores (2007) y adaptado por Valdivia y colaboradores (2010) para el caso de la ZMCM, en esta sección se evaluará si hay externalidades (y/o efectos de congestión) en la creación de empleo a nivel de sector de actividad a largo de las zM. El propósito es evaluar si las actividades asociadas a la clase creativa desarrollan externalidades diferenciadas en relación con el resto de actividades económicas.

La propuesta econométrica para identificar lo anterior es la siguiente:

$$
\text { difemp }_{\text {sec tor_actividad }}=a C l+b C l^{2}+c E d u c a c i o ́ n+e
$$

donde difemp es la diferencia en el nivel de empleo entre el año 2003 y 2008 a nivel de clase de actividad (del SCIAN) en una zM, $C l=\frac{E_{i,}}{A}, E$ es el empleo del sector en el año 2003 en el área $i, A$ representa la superficie de la zona metropolitana $i\left(\mathrm{en}^{\mathrm{kms}}{ }^{2}\right.$ ). $C I$ captura las externalidades positivas asociadas a las economías de aglomeración. $C I^{2}$ rescata los posibles efectos de "congestión” que puede generar una concentración elevada de firmas; Educación es

${ }^{13}$ Es importante señalar aquí que las externalidades que se analizarán en esta sección son las que operan en la creación de empleo y son de tipo Arrow-Marshall-Romer en el sentido de que operan intrasectorialmente. Sin embargo, en el contexto de la discusión de la clase creativa, las externalidades más emblemáticas son de tipo Jacobs y están más asociadas a la difusión de ideas o transmisión de conocimiento y que precisamente trascienden a los sectores y operan en el ámbito de la ciudad. Este último tipo de análisis está fuera del alcance de esta investigación. 
una variable proxy de capital humano y que en este trabajo se concibe como los años promedio de escolaridad de la población en el año 2005; $a, b$ y $c$ son parámetros por estimar. Las hipótesis centrales son las siguientes: 1) si $a$ es positivo, existen externalidades positivas en el empleo; y 2) si además $b$ es negativo, existen efectos de congestión en las externalidades (o si $b$ es diferente de cero o es positivo, no existen efectos de congestión).

El cuadro 5 muestra las estimaciones del modelo propuesto para identificar externalidades y posibles efectos de congestión en la creación de empleo a nivel de zM. En el ejercicio se calculan las densidades de empleo de acuerdo con la superficie total de la respectiva zm. ${ }^{14}$ En primera instancia se observa que la mayoría de los sectores de servicios despliegan un coeficiente positivo significativo en alguna de las variables de densidad de empleo — sugiriendo con ello derramas en empleo-. En particular, los sectores 56 (apoyo de negocios) y 72 (hoteles y restaurantes) muestran un coeficiente positivo significativo en $C I$, pero negativo en $C I^{2}$, lo que indica que este grupo de clases de actividad muestran efectos de congestión en las zonas metropolitanas.

Pero lo anterior no ocurre en el sector 54 (y tampoco en el sector 61 y 71), lo que podría implicar que estos sectores creativos todavía no están lo suficientemente desarrollados en las zm como los están otros sectores de servicios asociados al sector creativo, por lo que aún no están generando efectos de congestión en la creación de empleo. Estos resultados deben a su vez verse a la luz de los encontrados en la sección 4, donde se midió la centralización del empleo y se encontró que precisamente estos sectores creativos son los que están más concentrados (a nivel AGEB) respecto del centro económico de las zM.

El cuadro 5 también muestra los resultados para el sector manufacturero (31), y se observa que el coeficiente asociado a $C I$ es negativo y significativo, lo que sugiere la presencia de externalidades negativas al interior de las zonas metropolitanas. Este resultado es consistente con la tendencia de "desindustrialización" observada en las principales zm del país.

${ }^{14}$ En un ejercicio paralelo se realizaron las estimaciones considerando el promedio de las densidades de empleo a nivel de AGEB. Dichas estimaciones no se presentan en este documento pero están a disposición del lector previa comunicación con el autor. 


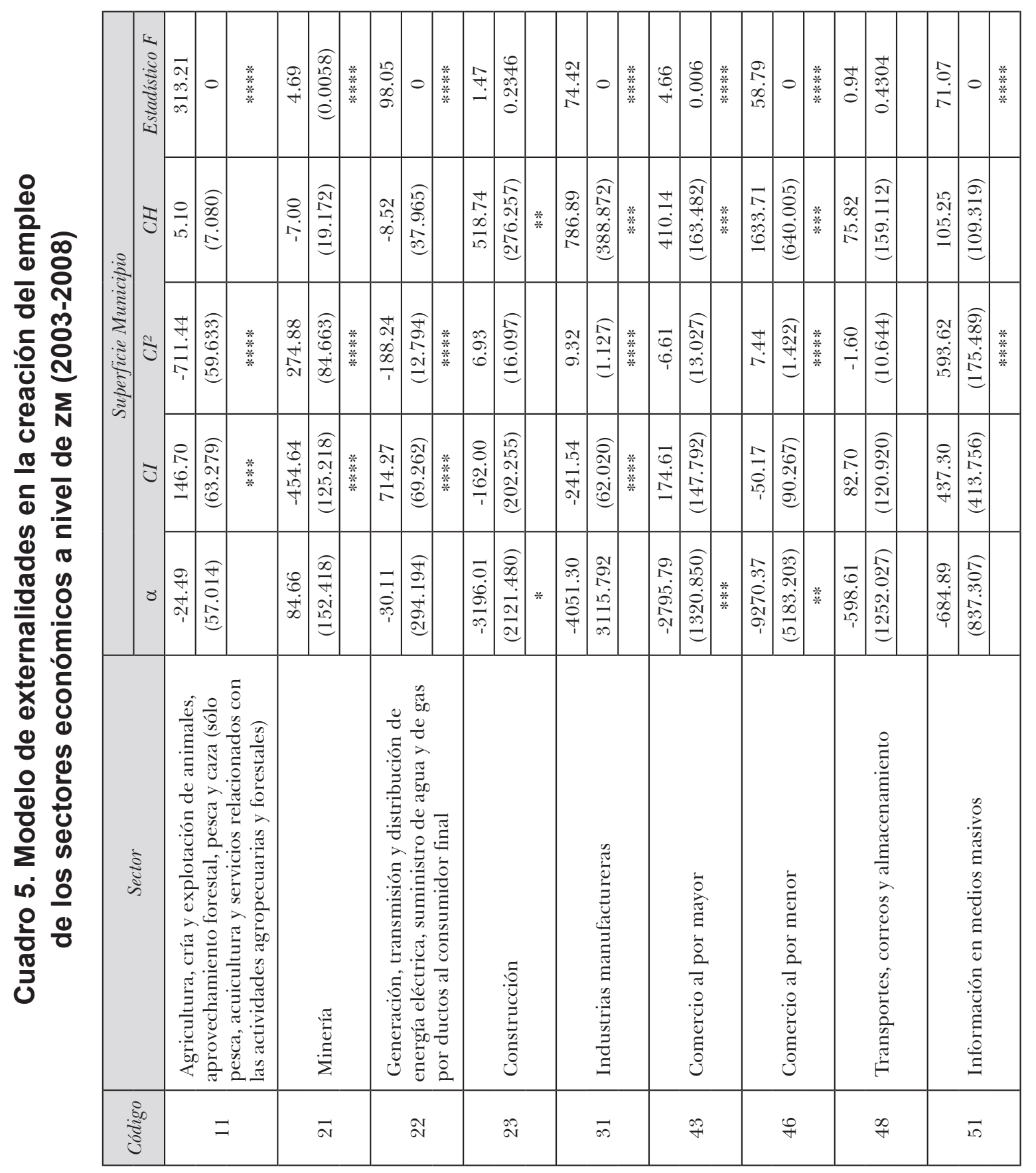




\begin{tabular}{|c|c|c|c|c|c|c|c|c|c|c|c|c|c|c|c|c|c|c|c|c|}
\hline 0 & 0 莡 & : & 0 & 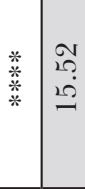 & 0 & * & 0 & \begin{tabular}{l|l}
$*$ \\
$*$ \\
$*$
\end{tabular} & 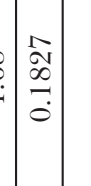 & $\mid \begin{array}{l}0 \\
0 \\
0 \\
0\end{array}$ & $0 \%$ & 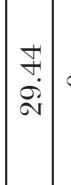 & ○ & $\left|\begin{array}{c}\infty \\
\stackrel{0}{+} \\
+\end{array}\right|$ & \begin{tabular}{l|l}
20 & $*$ \\
& $\%$ \\
0 & $*$
\end{tabular} & 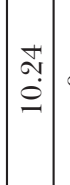 & $\circ \stackrel{*}{*}$ & $\mid \begin{array}{l}1 \\
0 \\
2 \\
10 \\
\end{array}$ & 0. & \\
\hline $\begin{array}{l}8 \\
1 \\
a \\
i \\
1\end{array}$ & 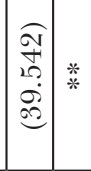 & $\begin{array}{l}\mathcal{V} \\
\mathcal{D} \\
\infty \\
\infty\end{array}$ & 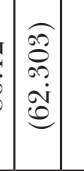 & $\begin{array}{l}5 \\
\sigma \\
\infty \\
\sigma \\
\sigma\end{array}$ & 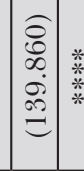 & 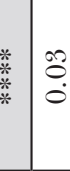 & 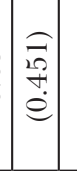 & $\mid \begin{array}{l}0 \\
20 \\
\infty \\
\infty \\
0 \\
0 \\
0\end{array}$ & 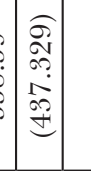 & 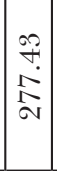 & 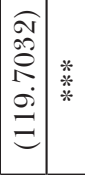 & $\begin{array}{l}20 \\
20 \\
20 \\
0 \\
0\end{array}$ & 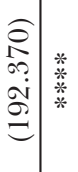 & $\left|\begin{array}{l}20 \\
0 \\
0 \\
0 \\
0\end{array}\right|$ & 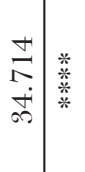 & $\begin{array}{c}+1 \\
0 \\
0 \\
0 \\
\infty \\
\infty \\
\end{array}$ & 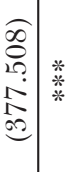 & \begin{tabular}{|l|}
10 \\
0 \\
0 \\
2 \\
1 \\
1
\end{tabular} & 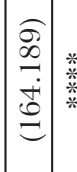 & \\
\hline $\begin{array}{l}\mathcal{O} \\
\sigma \\
2 \\
\mathscr{F} \\
F\end{array}$ & 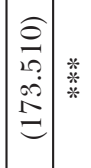 & $\begin{array}{l}0 \\
\infty \\
0 \\
0 \\
\sigma \\
\sigma \\
\sigma\end{array}$ & 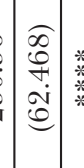 & 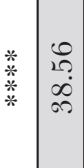 & $\mid \begin{array}{c}\widehat{F} \\
2 \\
\tilde{N} \\
\infty \\
= \\
=\end{array}$ & * & $\mid \begin{array}{l}\mathscr{Q} \\
0 \\
2 \\
\Xi \\
\Xi\end{array}$ & 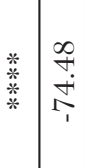 & 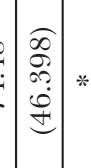 & 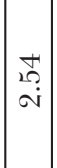 & \begin{tabular}{|c|}
20 \\
20 \\
20 \\
$\infty$ \\
$\infty$ \\
0 \\
0 \\
0
\end{tabular} & 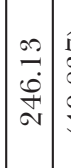 & 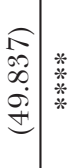 & 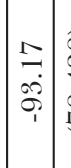 & 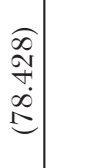 & $\mid \begin{array}{c}0 \\
0 \\
\hat{1} \\
\hat{v} \\
1\end{array}$ & 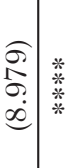 & 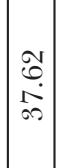 & 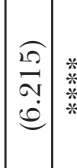 & \\
\hline 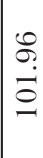 & 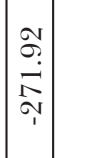 & \begin{tabular}{l} 
․ \\
$\hat{1}$ \\
0 \\
$\infty$ \\
\hdashline \\
\end{tabular} & 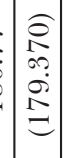 & $\begin{array}{l}20 \\
0 ? \\
0 \\
0 \\
0\end{array}$ & 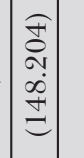 & $\begin{array}{l}10 \\
\frac{10}{80} \\
7 \\
1\end{array}$ & 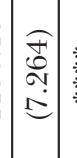 & 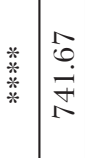 & 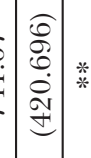 & \begin{tabular}{|c|}
$\infty$ \\
0 \\
10 \\
$\infty$
\end{tabular} & 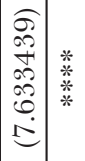 & 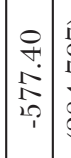 & 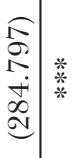 & $\begin{array}{l}\infty \\
\infty \\
\infty \\
\dot{g} \\
\stackrel{2}{=}\end{array}$ & 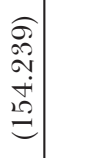 & $\begin{array}{l}+ \\
\infty \\
\dot{\mathbb{N}} \\
\mathbb{N}\end{array}$ & 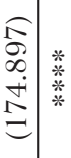 & $\mid \begin{array}{l}10 \\
1 \\
0 \\
0 \\
7 \\
7\end{array}$ & 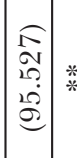 & \\
\hline 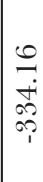 & 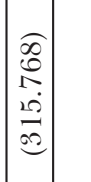 & $\begin{array}{l}\infty \\
0 \\
0 \\
0 \\
0 \\
0\end{array}$ & 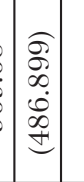 & 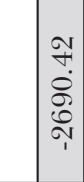 & 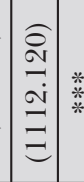 & * & $\begin{array}{l}\widehat{\sigma} \\
\infty \\
2 \\
2 \\
0 \\
0\end{array}$ & $\begin{array}{l}\text { क } \\
\text { aे } \\
\text { के } \\
\text { के } \\
\text { vै }\end{array}$ & 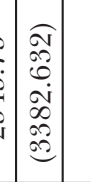 & 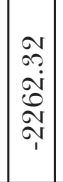 & 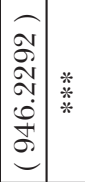 & 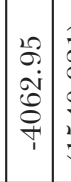 & 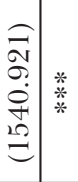 & $\begin{array}{ll}0 \\
0 \\
0 \\
0 \\
0 \\
0 \\
1 & 1 \\
1 & 1 \\
& \end{array}$ & 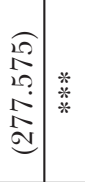 & \begin{tabular}{|c|}
8 \\
0 \\
0 \\
28 \\
10 \\
20 \\
1
\end{tabular} & 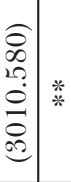 & $\begin{array}{l}0 \\
0 \\
0 \\
0 \\
0 \\
\stackrel{2}{+}+1 \\
1\end{array}$ & 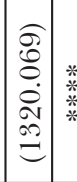 & \\
\hline & 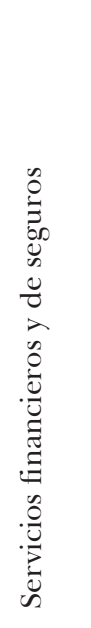 & & 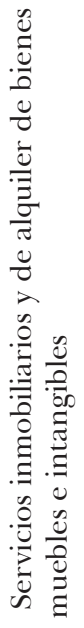 & & 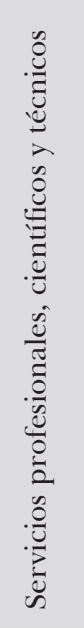 & & 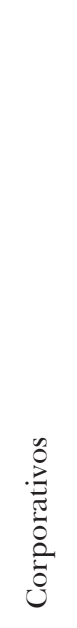 & & 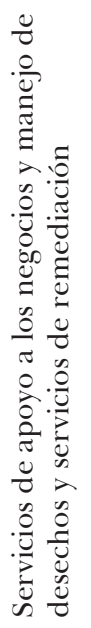 & & 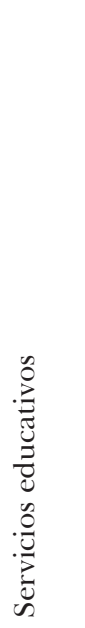 & & 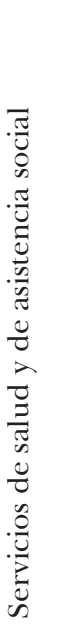 & & 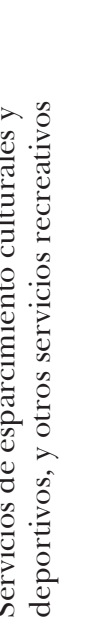 & & 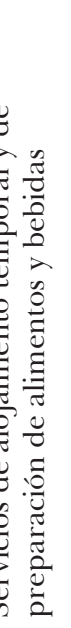 & & 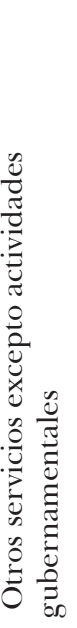 & \\
\hline & กิ & & in & & in & & 10 & & 10 & & $\overline{0}$ & & $\mathscr{J}$ & & K & & i & & $\vec{\infty}$ & \\
\hline
\end{tabular}




\section{Dependencia espacial de la clase 54 y clusters de la clase creativa al interior de las zonas metropolitanas}

Hasta ahora se ha mostrado que el sector 54 y sectores asociados $(56,61$, 72) despliegan los mayores niveles de concentración de empleo y de generación de derramas (de empleo) en comparación con el resto de los sectores económicos, para las 56 zM. En esta última sección nos enfocaremos ahora a analizar el interior de las zonas metropolitanas para apreciar cómo las características de centralidad (y posible generación de derramas) de los sectores creativos, se asocian a patrones de dependencia espacial.

Para llevar a cabo este análisis se seleccionaron las $8 \mathrm{zm}$ (del conjunto de 56), que reportaron los niveles más altos de participación de empleo del sector 54 (ver figura 1). Es importante resaltar que este grupo de ciudades es una mezcla de zm grandes como la Ciudad de México, Monterrey y Guadalajara, y zm de medio tamaño como Querétaro, Cancún, Villahermosa y Córdoba.

Se procedió a calcular un índice de Moran a escala de AGEB (cuadro 7), considerando tres variables del sector 54: 1) logaritmo (log) de la productividad laboral; 2) log del VACB; y 3) log del empleo del sector respecto del total del empleo. El cálculo se basa en una matriz de pesos espaciales que considera los 4 AgEbs más cercanos a cada AGEB. Del resultado obtenido, lo primero que se observa es que todos los índices son elevados y estadísticamente significativos, lo que indica una fuerte autocorrelación espacial de las variables para el conjunto de las 8 zM; no obstante, las zM de Mérida y Guadalajara despliegan los índices más elevados bajo el criterio de interacción espacial seleccionado (4 más cercanos).

Como es conocido en la literatura de estadística espacial, la dependencia espacial global que despliega el índice de Moran tiene una descomposición a nivel local (Anselin, 1995), lo cual nos permite identificar dónde se localizan los hot spots de concentración de empleo del sector 54 al interior de las zM consideradas a nivel de АGEв. En los mapas de la figura 5 se muestra el despliegue cartográfico de los indicadores locales de autocorrelación espacial (llamadas LISA por sus siglas en inglés) en las zM escogidas para el año 2008. Las agrupaciones en negro indican que la proporción de empleo del sector 54 en los AgEBs (que forman ese conglomerado), se encuentra estadística y significativamente por arriba del promedio de la 


\section{Cuadro 6. Dependencia espacial en diversos indicadores del sector 54 en las ZM creativas del país en 2008}

\begin{tabular}{|c|c|c|c|c|}
\hline Cve_ZM & Nombre zona metropolitana & Log productividad & $\log V A C B$ & $\begin{array}{c}\text { Log participación } \\
\text { empleo }\end{array}$ \\
\hline \multirow{2}{*}{13} & \multirow{2}{*}{ Valle de México } & 0.3013 & 0.4137 & 0.4776 \\
\hline & & $* * * *$ & $* * * *$ & $* * * *$ \\
\hline \multirow{2}{*}{36} & \multirow{2}{*}{ Querétaro } & 0.3827 & 0.4445 & 0.457 \\
\hline & & $* * * *$ & $* * * *$ & $* * * *$ \\
\hline \multirow{2}{*}{31} & \multirow{2}{*}{ Monterrey } & 0.3705 & 0.4551 & 0.5039 \\
\hline & & $* * * *$ & $* * * *$ & $* * * *$ \\
\hline \multirow{2}{*}{41} & \multirow{2}{*}{ Villahermosa } & 0.3804 & 0.4843 & 0.5703 \\
\hline & & $* * * *$ & $* * * *$ & $* * * *$ \\
\hline \multirow{2}{*}{55} & \multirow{2}{*}{ Mérida } & 0.4727 & 0.5783 & 0.653 \\
\hline & & $* * * *$ & $* * * *$ & $* * * *$ \\
\hline \multirow{2}{*}{53} & \multirow{2}{*}{ Córdoba } & 0.2772 & 0.3582 & 0.3975 \\
\hline & & $* * * *$ & $* * * *$ & $* * * *$ \\
\hline \multirow{2}{*}{37} & \multirow{2}{*}{ Cancún } & 0.3377 & 0.4552 & 0.5481 \\
\hline & & $* * * *$ & $* * * *$ & $* * * *$ \\
\hline \multirow{2}{*}{21} & \multirow{2}{*}{ Guadalajara } & 0.4449 & 0.5577 & 0.6272 \\
\hline & & $* * * *$ & $* * * *$ & $* * * *$ \\
\hline
\end{tabular}

Nota: Se calcularon los índices de Morán a nivel de AGEB al interior de cada una de las zm analizadas. Todos los índices generaron pseudo p valores cercanos al 0.0001 y son señalados en el cuadro con ****. Las pruebas se realizaron bajo un criterio de permutación computacional para generar la distribución experimental con la cual se realizaron las pruebas de inferencia. Se consideró una matriz de pesos espaciales con los 4 agebs más cercanos.

ZM y además el promedio de la participación del empleo de la 54 en los 4 AGEBS más cercanos también está estadística y significativamente por arriba del promedio de la ZM; en conjunto, los AGEBS en negro forman el cluster creativo en la ZM y que dista de ser una aglomeración aleatoria.

El principal resultado a destacar de los mapas es que hay clusters bien definidos al interior de las ZM (con alta participación del sector 54) y además dichos clusters, en general, coinciden con las cercanías al centro económico de la ZM (considerado éste de manera a priori como la plancha del zócalo o la plaza central). Por ejemplo, obsérvese en la figura de la ZMCM cómo el cluster de la industria creativa se ubica en el área centro-poniente de la ciudad cercana al corredor Insurgentes sur-centro y de Reforma; mientras que en la ciudad de Guadalajara el cluster creativo se localiza en la parte norponiente. 
Figura 5a. Zona Metropolitana de Mérida a nivel de AGEB e identificación espacial de su aglomeración creativa

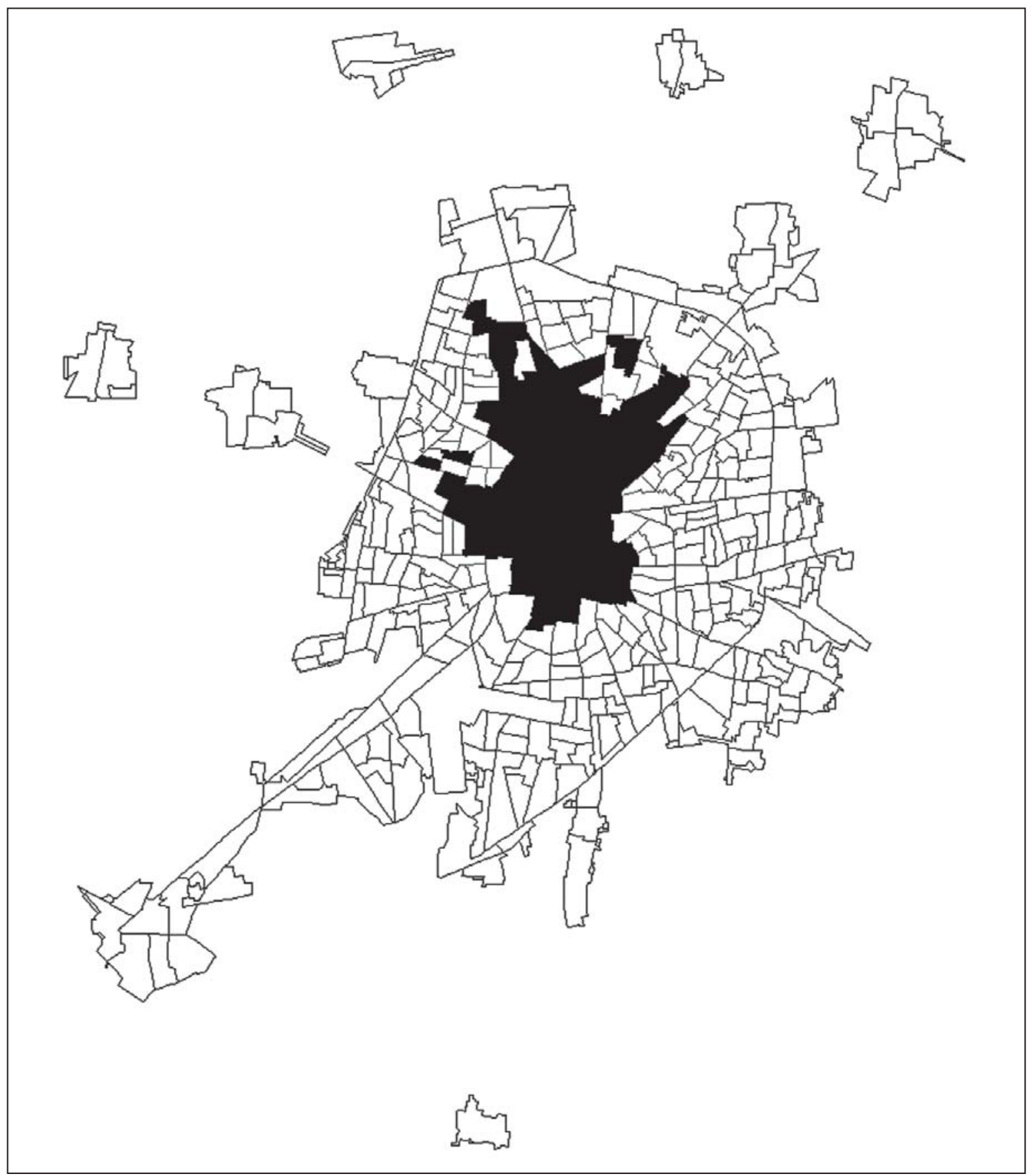

Nota: Se identificó la aglomeración creativa a partir de indicadores locales de autocorrelación espacial. 
Figura 5b. Zona Metropolitana de Córdova a nivel de AGEB e identificación espacial de su aglomeración creativa

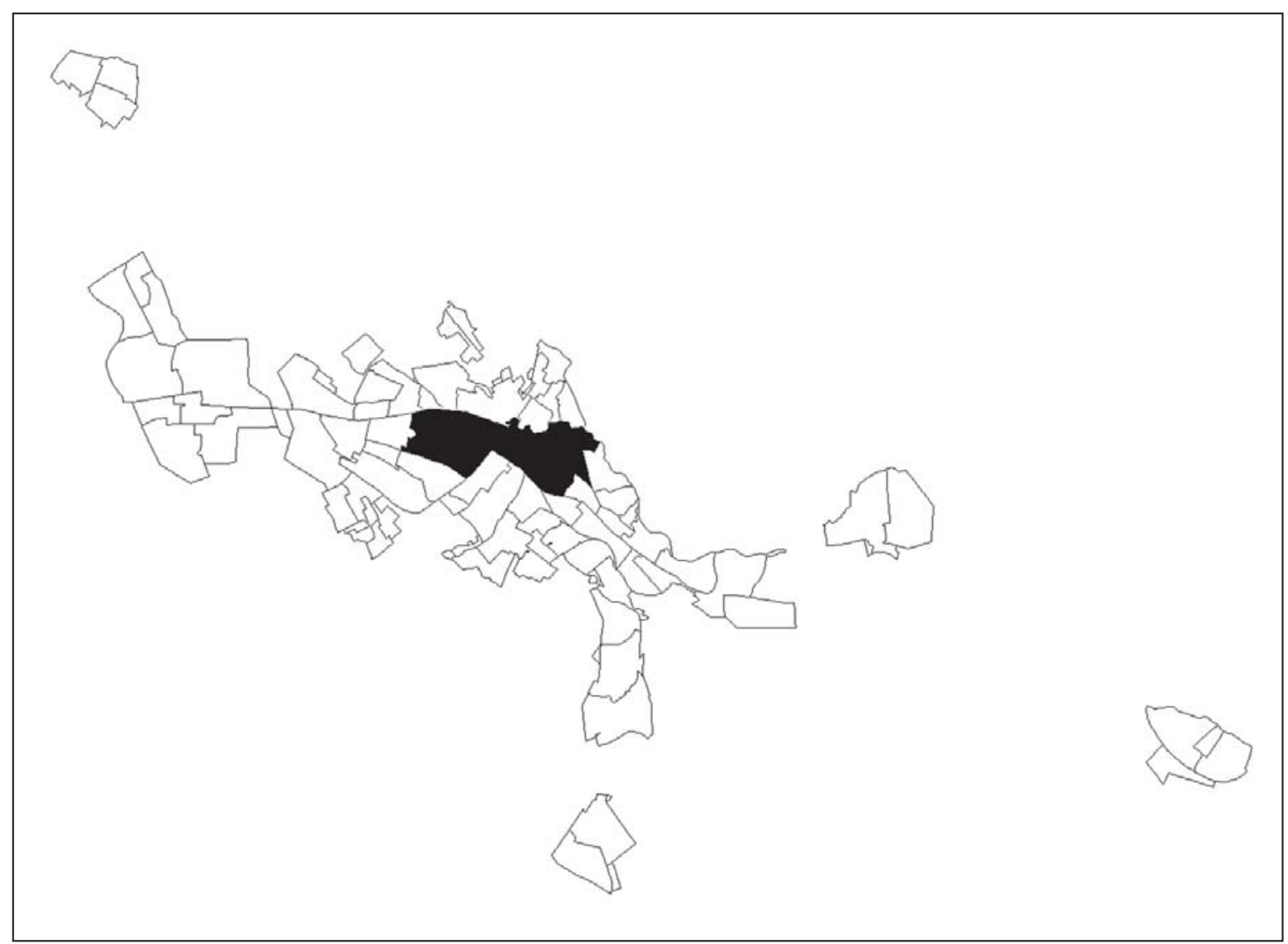

Nota: Se identificó la aglomeración creativa a partir de indicadores locales de autocorrelación espacial. 
Figura 5c. Zona Metropolitana de Cancún a nivel de AGEB e identificación espacial de su aglomeración creativa

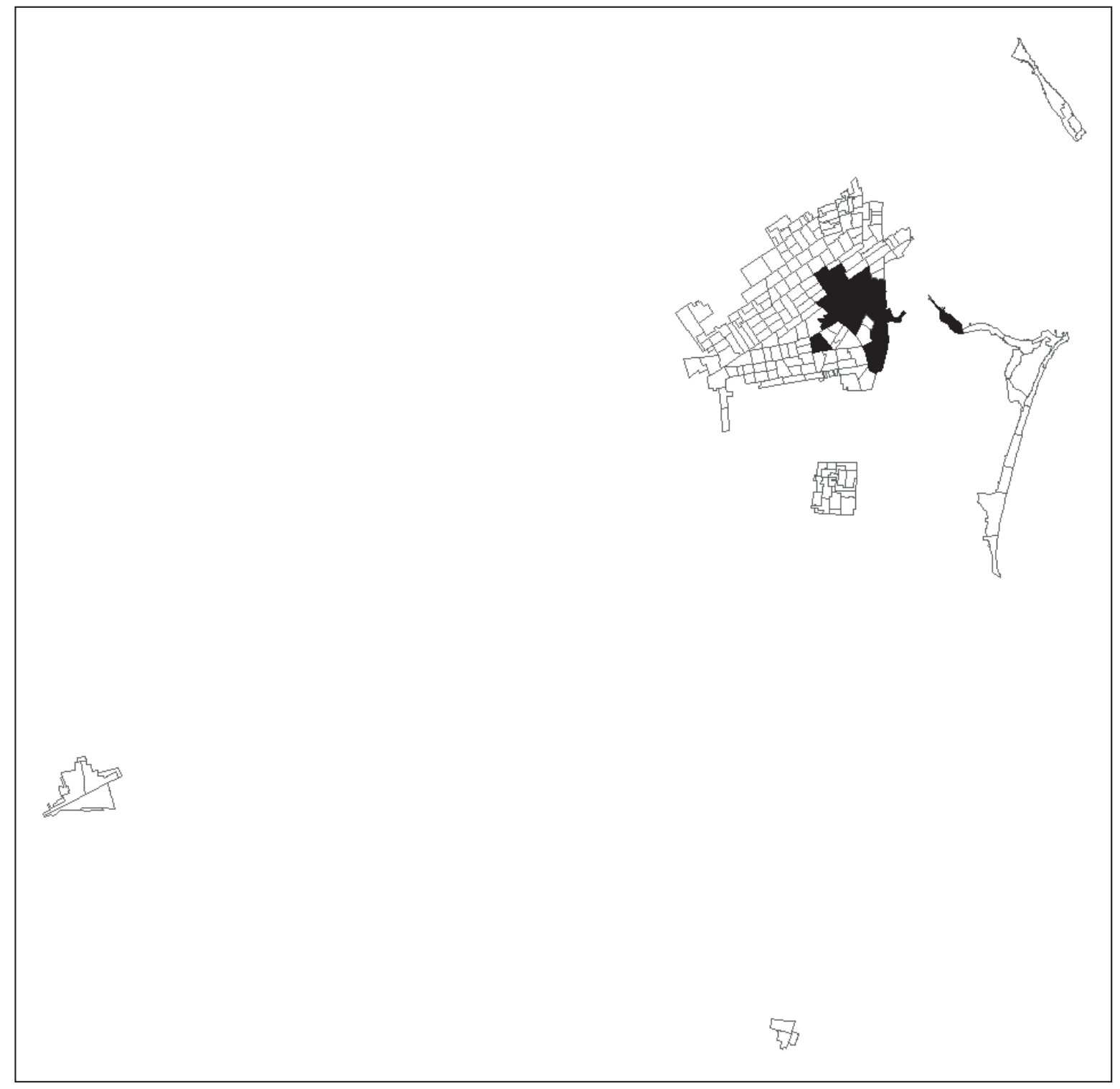

Nota: Se identificó la aglomeración creativa a partir de indicadores locales de autocorrelación espacial. 
Figura 5d. Zona Metropolitana de Guadalajara a nivel de AGEB e identificación espacial de su aglomeración creativa

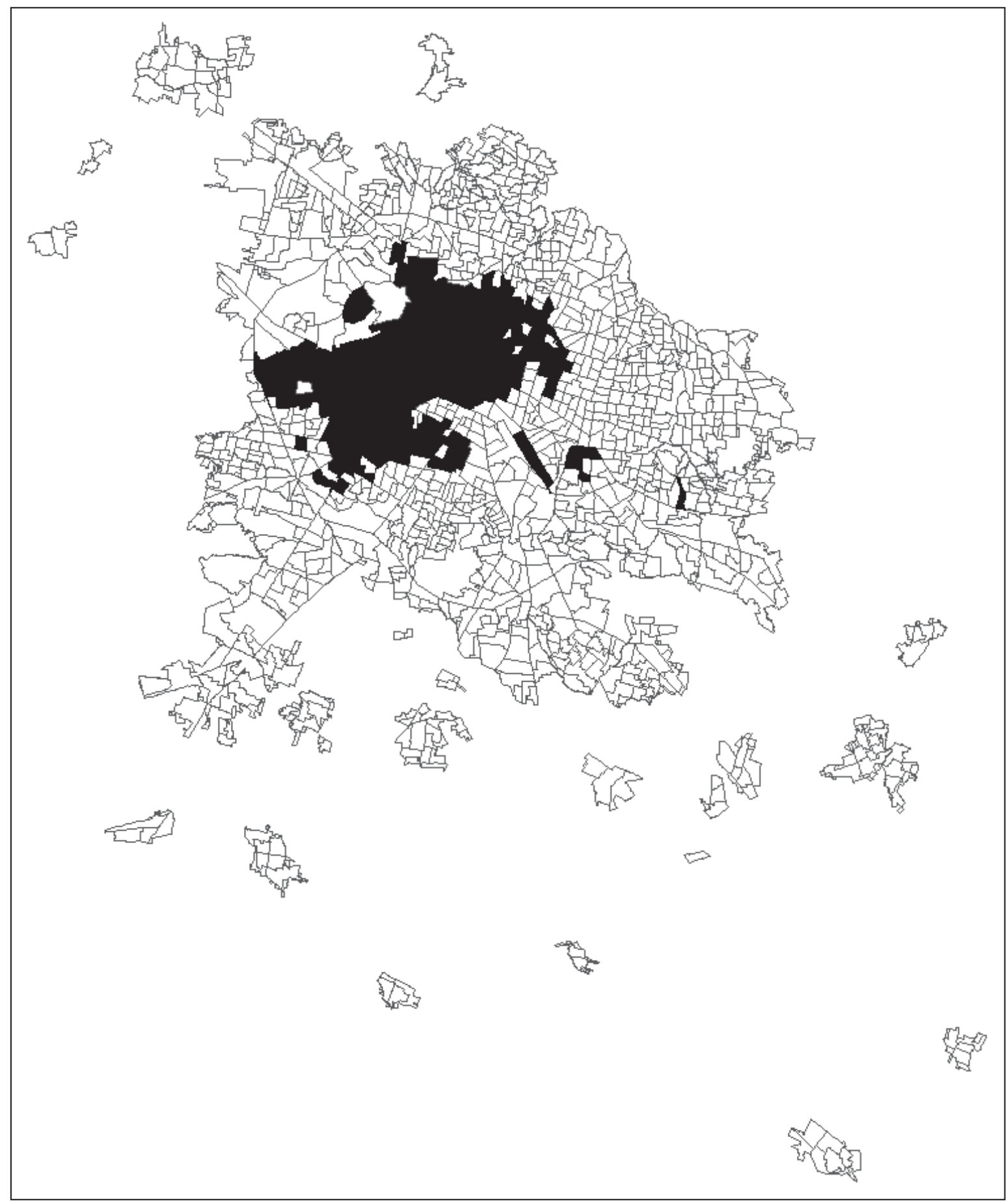

Nota: Se identificó la aglomeración creativa a partir de indicadores locales de autocorrelación espacial. 
Figura 5e. Zona Metropolitana de la Ciudad de México a nivel de AGEB e identificación espacial de su aglomeración creativa

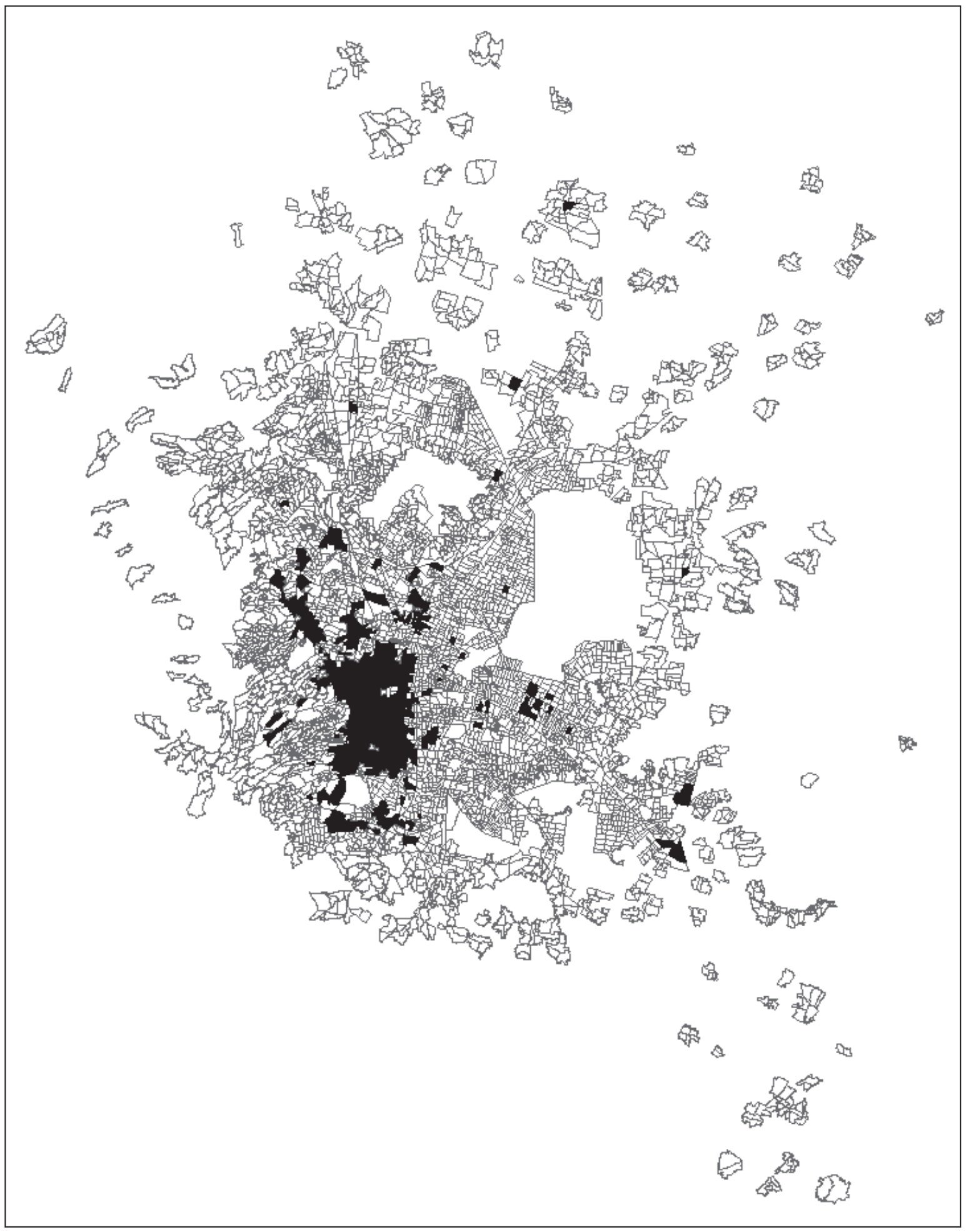

Nota: Se identificó la aglomeración creativa a partir de indicadores locales de autocorrelación espacial. 
Figura 5f. Zona Metropolitana de Querétaro a nivel de AGEB e identificación espacial de su aglomeración creativa

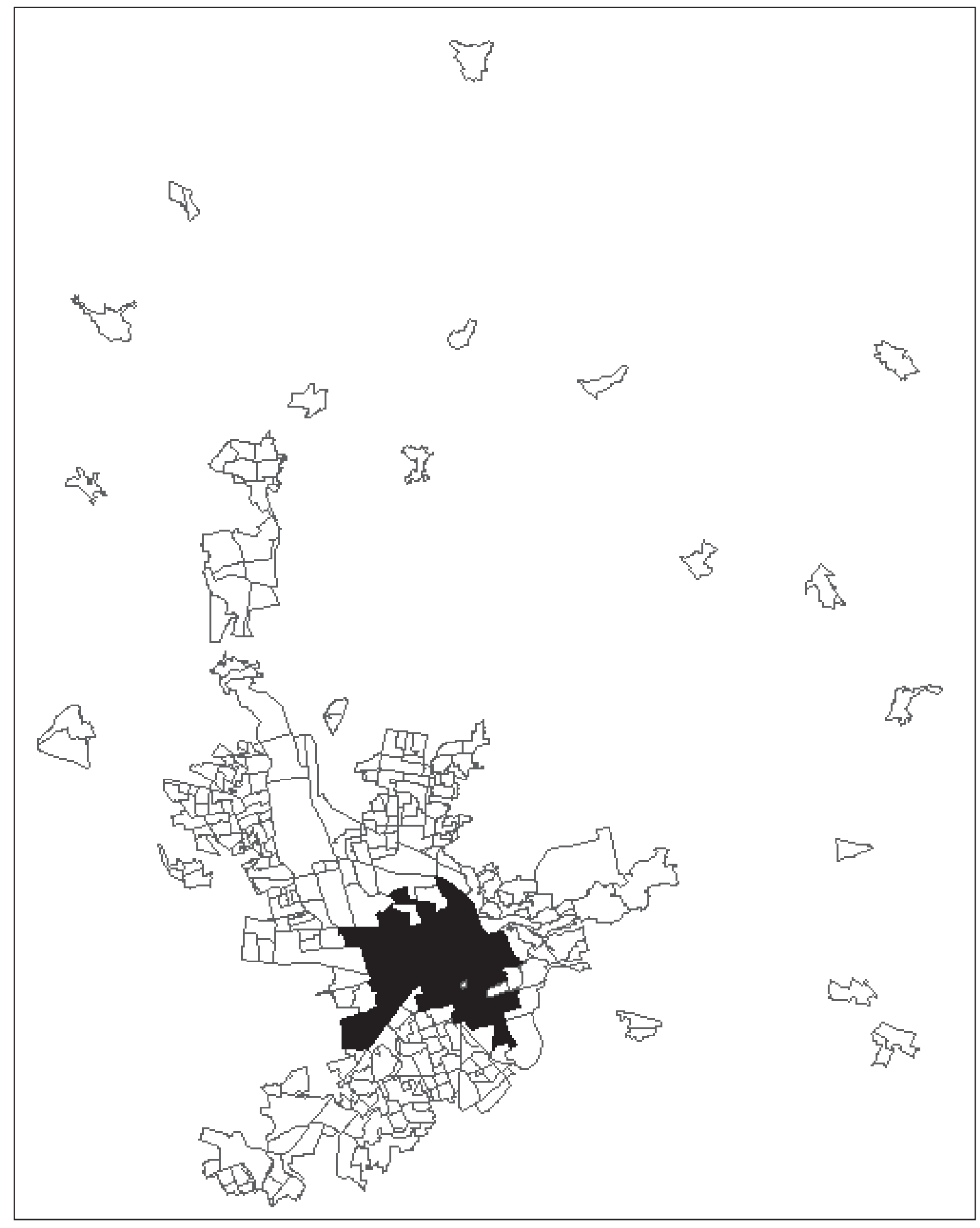

Nota: Se identificó la aglomeración creativa a partir de indicadores locales de autocorrelación espacial. 
Figura 5g. Zona Metropolitana de Villahermosa a nivel de AGEB e identificación espacial de su aglomeración creativa

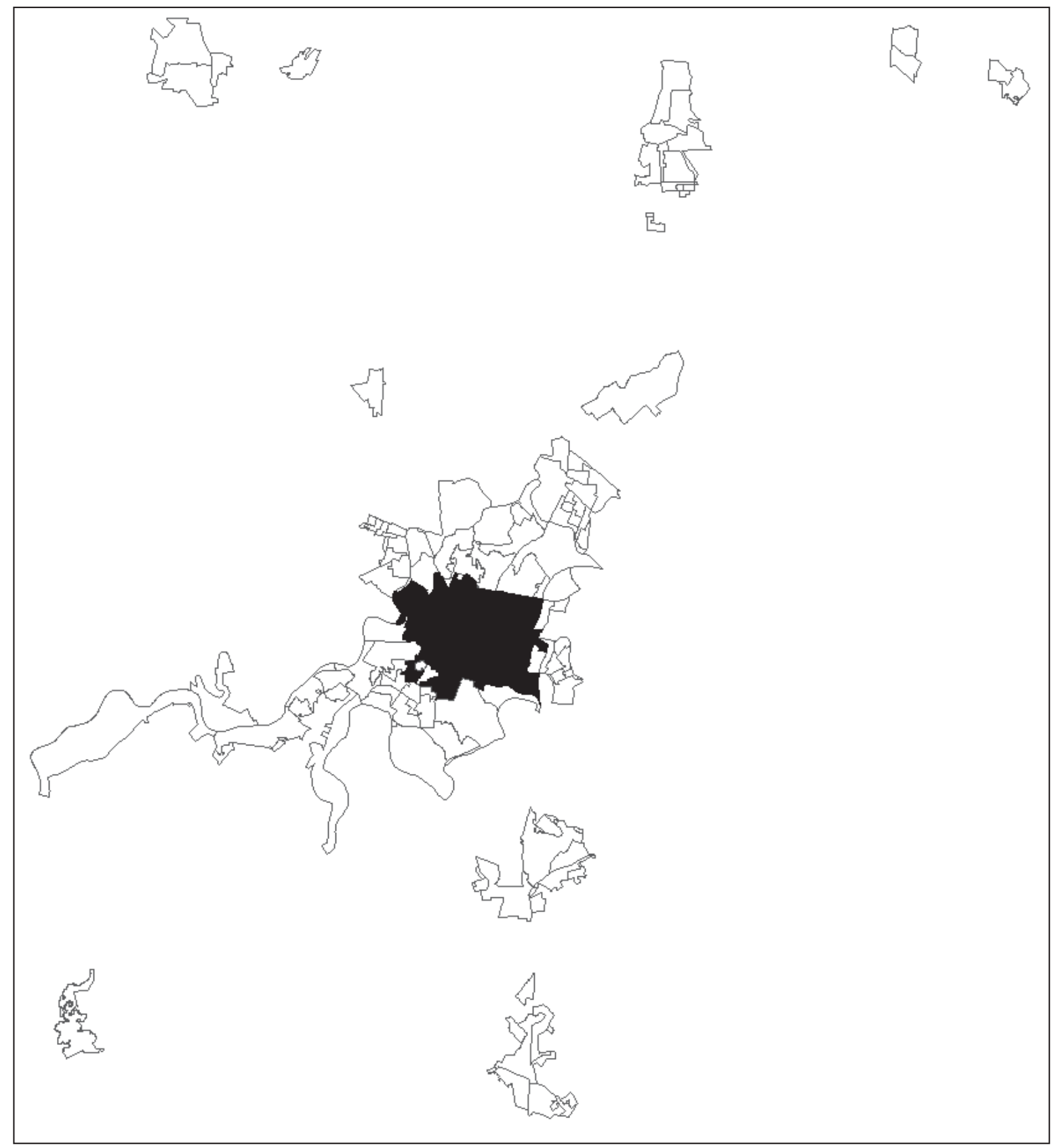

Nota: Se identificó la aglomeración creativa a partir de indicadores locales de autocorrelación espacial. 
Figura 5h. Zona Metropolitana de Monterrey a nivel de AGEB e identificación espacial de su aglomeración creativa

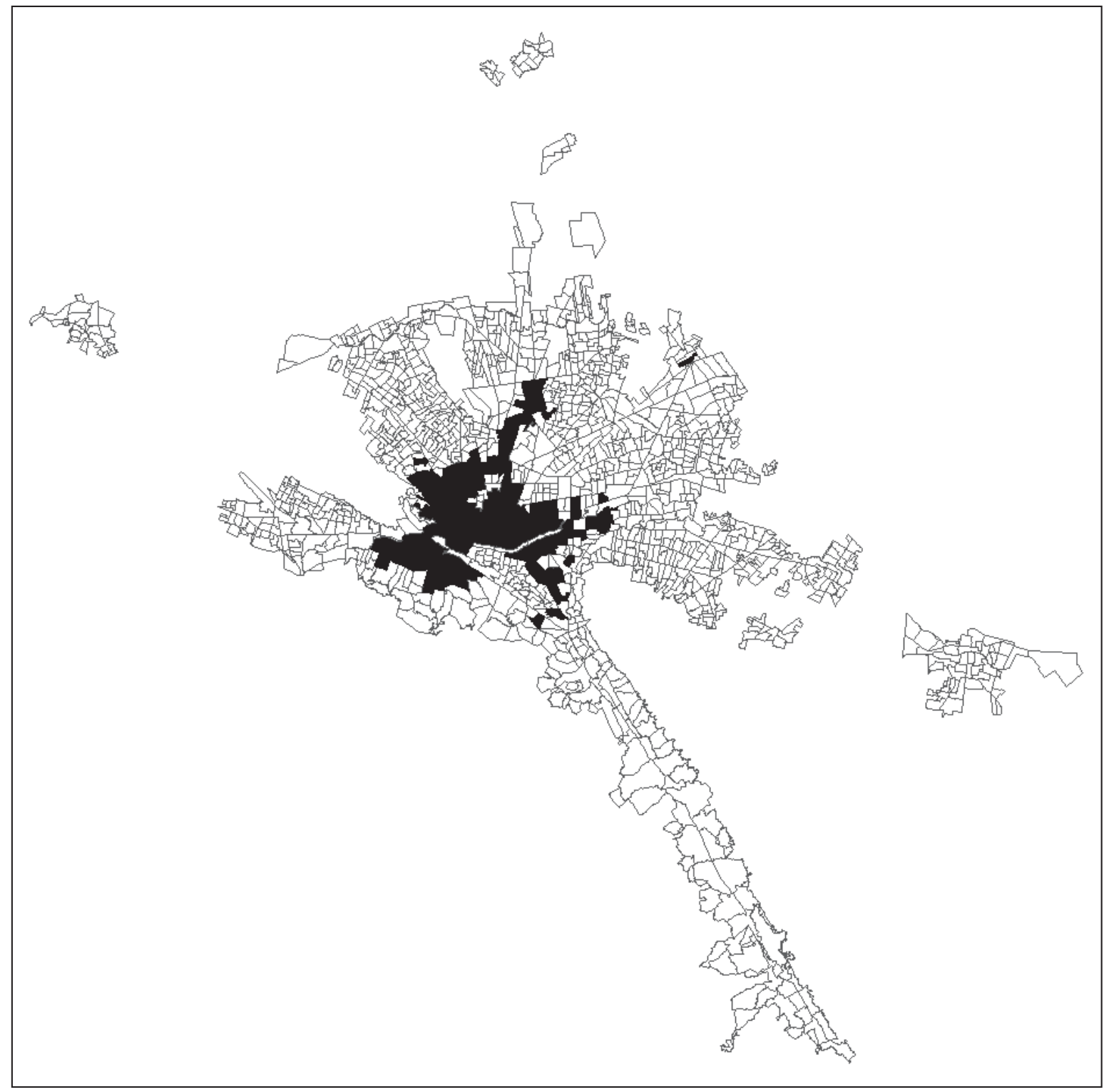

Nota: Se identificó la aglomeración creativa a partir de indicadores locales de autocorrelación espacial. 


\section{Conclusiones}

En este trabajo se concluye que las transformaciones recientes de la estructura urbana de las zonas metropolitanas de México han estado influenciadas por la consolidación de actividades económicas asociadas a la clase creativa. En particular, nuestros resultados indican que estas actividades creativas influyen positivamente en la dinámica de crecimiento de las zM y además, entre todas las actividades, son las que despliegan tanto mayor concentración espacial del empleo en las cercanías de los centros económicos de las ZM, como mayor capacidad para generar derramas en la creación de empleo.

Los resultados de la investigación sugieren que el crecimiento de las zonas metropolitanas en años recientes y de su eventual convergencia no depende sólo de la presencia de un stock de capital humano, sino también es necesario que exista un sector de industrias creativas (o de vinculación) al interior de las ciudades. Este elemento es fundamental porque se convierte en el pivote por el cual las zM podrán ser capaces de retener y atraer gente calificada. Indudablemente hay grandes disparidades regionales en esta dinámica, pues contrastan zm como la Ciudad de México y Querétaro donde se encuentran los niveles más altos de empleo en actividades creativas frente a zM como Minatitlán o Tula - dependientes de la refinación del petróleo- que tienen los niveles más bajos. Por lo encontrado en esta investigación, el reto para estas últimas ZM es, desde una perspectiva de política regional, generar una estrategia para dotar a estas zonas urbanas de una base de actividades creativas con la cual se pueda repercutir positivamente a la economía local.

En décadas recientes, la globalización provocó que importantes ciudades en diversas regiones del mundo - por ejemplo, Nueva York, Londres y Tokio, entre otras- perdieran sus ventajas como nodos manufactureros y tuvieran rápidamente que transformarse en ciudades especializadas en "producción de ideas" (Glaeser, 2011). Los resultados de esta investigación sugieren, guardando toda proporción, que estos cambios empiezan a presentarse en diversas zM de tamaño grande (Ciudad de México) y medio (Querétaro), y seguramente esta tendencia se reforzará en el futuro inmediato a lo largo del sistema de ciudades. Como señalamos en la sección 6, las actividades económicas que detectamos 
como asociadas a las industrias creativas no sólo están generando aglomeraciones espaciales y externalidades positivas, sino que a diferencia de otros sectores (i.e., manufactura), están lejos de desarrollar dinámicas de congestión en las zm. Esto último puede ser un indicador del espacio que estas actividades pueden todavía alcanzar para su crecimiento dentro de las ciudades en México.

En esta investigación solamente realizamos una aproximación de la influencia que tienen las actividades económicas creativas en la dinámica de crecimiento intrametropolitano. Sin embargo, no analizamos el componente de movilidad poblacional, y en particular de la migración calificada, en esta dinámica de consolidación de una clase creativa al interior de las zM; asimismo, tampoco analizamos el componente ocupacional —en vez del sectorial- de la clase creativa. Estos últimos pendientes tendrán que ser analizados en una futura investigación.

\section{Bibliografía}

Anselin, L. (1995). Local Indicators of Spatial Association-LISA. Geographical Analysis, 27(2), 93-113.

Asuad, N. y Quintana, L. (2010). Crecimiento económico, convergencia y concentración económica espacial en las entidades federativas de México 1970-2008. Investigaciones Regionales, 18, 83-106.

Barro, R. y Sala-i-Martin, X. (1992). Convergence. Journal of Political Economy, $100(2), 223-51$.

Baumol, W. (1986). Productivity growth, convergence, and welfare: What the long-run data show. American Economic Review, 76(5), 1072-1085.

Boix, R. y Lazzeretti, L. (2012). Las industrias creativas en España: Una panorámica. Investigaciones Regionales, 22, 181-206.

Boschma, R. y Fritsch, M. (2009). Creative class and regional growth: Empirical evidence from seven European countries. Economic Geography, 85(4), 391-423.

Brakman, S., Garretsen, H. y Marrewijk, C. V. (2009). The new introduction to geographical economics. Reino Unido: Cambridge University Press.

Calderón, C. y Tykhonenko, A. (2007). Convergencia regional e inversión extranjera en México en el contexto del TLCAN, 1994-2002. Investigación Económica, 66(259), 9-41.

Esquivel, G. (1999). Convergencia regional en México, 1940-1995. El Trimestre Económico, 66(264), 725-761. 
Fingleton, B., Igliori, D., Moore, B. y Odedra, R. (2007). Employment growth and cluster dynamics. En K. Polenske (Ed.), The Economic Geography of Innovation (pp. 60-84). Cambridge: Cambridge University Press.

Florida, R. (2012). The rise of the creative class. 10th anniversary edition-revised and expanded (1ra. ed.). Nueva York: Basic Books.

Florida, F., Mellander, C., Stolarick, K. y Ross, A. (2012). Cities, skills and wages. Journal of Economic Geography, 12(2), 355-377.

Florida, R. (2005). Cities and the creative class. Nueva York: Routledge.

Garza, G. (Coord.). (2006). La organización espacial del sector servicios en México: El Colegio de México.

Glaeser, E. (2005). Review of Richard Florida's The rise of the creative class. Regional Science and Urban Economics, 35(5), 593-596.

Glaeser, E. (2008). Cities, agglomeration, and spatial equilibrium. Nueva York: Oxford University Press.

Glaeser, E. (2011). Triumph of the city. Nueva York: The Penguin Press.

Glaeser, E. y Khan, M. (2001). Decentralized employment and the transformation of the American city. Brookings-Wharton Papers on Urban Affairs, 1-63.

Instituto Nacional de Estadística y Geografía (INEGI) (2001). XII Censo General de Población y Vivienda, 2000. Aguascalientes, México: Instituto Nacional de Estadística y Geografía.

Instituto Nacional de Estadística y Geografía (INEGI) (2004). Censos Económicos, 2004. Aguascalientes, México: Instituto Nacional de Estadística y Geografía.

Instituto Nacional de Estadística y Geografía (INEGI) (2009). Censos Económicos, 2009. Aguascalientes, México: Instituto Nacional de Estadística y Geografía.

Instituto Nacional de Estadística y Geografía (INEGI) (2011). Censo de Población y Vivienda, 2010. Aguascalientes, México: Instituto Nacional de Estadística y Geografía.

Krätke, S. (2010). Creative cities and the rise of the dealer class: A critique of Richard Florida's approach to urban theory. International Journal of Urban and Regional Research, 34(3), 835-53.

Kirdar, M. y Saracoglu, D. (2008). Migration and regional convergence: An empirical investigation for Turkey. Papers in Regional Science, 87(4), 545-566.

Martin-Brelot, H., Grossetti, M., Eckert, D., Gritsai, O. y Kovács, Z. (2010). The spatial mobility of the 'creative class': A European perspective. International Journal of Urban and Regional Research, 34(4), 854-70.

Méndez, R., Michelini, J., Prada, J. y Tébar, J. (2012). Economía creativa y desarrollo urbano en España: Una aproximación a sus lógicas espaciales. EURE, 38(133), 5-32.

Mendoza, M. A. (2013). Externalidades del capital humano y espaciales, su influencia en el crecimiento de las ciudades en México. En M. Valdivia y J. Delgadillo (Coords.), La geografía y la economía en sus vínculos actuales (pp. 
221-232). México: Universidad Nacional Autónoma de México, Centro Regional de Investigaciones Multidisciplinarias, Instituto de Investigaciones Económicas.

Moretti, E. (2012). The new geography of jobs. Boston: Houghton Mifflin Harcourt. Secretaría de Desarrollo Social (Sedesol), Consejo Nacional de Población (Conapo), Instituto Nacional de Estadística y Geografía (INEGI) (2007). Delimitación de zonas metropolitanas de México 2005. México: Autor.

Richardson, H. (1993). Modelos en torno a la estructura urbana. En S. Flores (Coord.), Desarrollo metropolitano. Análisis y perspectivas (lecturas sobre la teoría y el desarrollo metropolitano (pp. 25-34). México: Benemérita Universidad Autónoma de Puebla.

Storper, M. y Scott, A. (2009). Rethinking human capital, creativity and urban growth. Journal of Economic Geography, 9(2), 147-167.

Valdivia, M., Delgadillo, J. y Galindo, C. (2010). Nuevos patrones espaciales en las derramas de empleo en la zona metropolitana de la Ciudad de México. Revista Problemas del Desarrollo, 163(41), 99-117. 